\title{
Three Cases That Demonstrate How Students Connect the Domains of Mathematics and Computing
}

\author{
Odd Petter Sand ${ }^{1}$, Elise Lockwood ${ }^{3}$, Marcos D. Caballero ${ }^{1,2}$, and Knut Mørken ${ }^{1}$ \\ ${ }^{1}$ Centre for Computing in Science Education (CCSE), University of Oslo, N-0316 Oslo, Norway \\ ${ }^{2}$ Department of Physics and Astronomy, Department of Computational Mathematics, Science and \\ Engineering, and CREATE for STEM Institute, Michigan State University, East Lansing, MI 48823 \\ ${ }^{3}$ Department of Mathematics, Oregon State University, Corvallis, OR 97331
}

This study uses actor-oriented transfer perspective to investigate different ways in which students make connections across the domains of mathematics and computing. We interview first-year students at the University of Oslo as they work with a set of tutorials that we designed to integrate knowledge from both domains. The cases we present here demonstrate four different types of cross-domain connections: (a) mathematically reproducing the work of a computer program, (b) cyclically improving a program to produce better output, (c) coupling math to output to justify program improvements and (d) coupling math to code to justify program design. We provide rich examples of the ways in which students make these connections and discuss affordances for mathematical learning in this context.

\section{Introduction}

The last several decades has seen computers take over more and more tasks that used to be the domain of the human mind alone. Already, there is a concern that while mathematics is at the core of what computers can do, "the omnipresent mathematics is mainly hidden in all sorts of apparatus, which function as black boxes for its users" (Gravemeijer et al., 2017, p. 53; see also Williams \& Wake, 2007). In mathematics classrooms, the worry is that students will become dependent on computational tools to do mathematics without understanding the underlying principles of either.

Nevertheless, computers and programming are becoming ever more important in the practice and teaching of mathematics (e.g., Broley et al., 2018; Passey, 2017). The reasons for this movement are manifold and include allowing for the investigation of more and different topics, giving students more hands-on experience, and the envisioned potential "to have mathematics come to feel more natural, relevant, and less intimidating" (diSessa, 2018, p. 25). However, we as a field are just beginning to understand how the integration of computing into math affects student thinking and learning. How does one domain connect to the other from the students' point of view, and what do these connections afford?

Our focus in this paper is to study the ways in which undergraduate students connect mathematics and computing. While studies of undergraduate students' mathematical work using computing do exist (see Section 2.2), there have been calls for more attention to be paid to computing in math education research (e.g., Lockwood \& Mørken, 2021). We therefore aim to provide several rich examples that demonstrate how working in a context where mathematics and computing are integrated can be beneficial for the students' thinking and learning about mathematics.

To that end, we first present our theoretical perspective and position our study within the literature, before formulating our research questions (Section 2.3). In Section 3, we describe the study design and the process of analysis, while Section 4 deals with the analytical framework we developed to classify connections. Finally, we present our cases (including brief descriptions of the tutorials we designed) and our analysis of each in Section 5 and discuss these results and their implications for teaching and further research in Section 6. 


\section{Theoretical Perspective and Background Literature}

\subsection{Theoretical Perspective}

Connections and their importance in the context of learning was highlighted as early as the $19^{\text {th }}$ century. Harald Høffding (1892) argued that "what matters is how the new situation is connected with the thinker's trace of a previous situation, which may be quite idiosyncratic" (cited in Lobato \& Siebert, 2002). While there are many different types of situations students may encounter as they work with computers in mathematics and science (see for instance Weintrop et al., 2016), the ways in which students connect their work on the computer with mathematical concepts is largely unexplored territory (the examples of previous work we found are cited in Section 2.2).

While this paper is not focused on transfer of learning per se, the actor-oriented transfer (AOT) perspective (Lobato, 2012; Lobato \& Siebert, 2002) provides us with a useful language to describe these connections. An important facet of the AOT framework is its focus on similarities as seen from the actors' ${ }^{1}$ point of view. These similarities allow actors to make connections between a situation and some previous situation or activity that they regard as similar and relevant. To Lobato and Siebert (2002), "[AOT] is defined as the personal construction of relations of similarity between activities, or how actors see situations as similar." This applies even when students make unexpected connections that may or may not result in incorrect performance and stands in contrast with the traditional views of transfer (Lobato, 2008). Note especially the focus in AOT on situations (for instance problems and techniques) being similar, as opposed to only connecting knowledge.

Connections may also be considered to be one of the three ways that students generalize, as in the framework developed by Ellis et al., (2017). In that framework, generalizing consists of relating (i.e., connecting), extending, and forming (deriving general results from particular results). This framework explicitly takes a cue from Lobato and uses an actor-oriented approach. When students relate situations, they either connect back from the current situation to a previous one or invent an analogy, imagining a new situation similar to the current one. Our cross-domain connections correspond to what Ellis et al. call intercontextual forms of generalizing, and their goal in studying them is the same as ours: "seeking to understand the processes by which students construct relations of similarity that they experience as meaningful." (p. 3)

The setting we are examining (see Section 3.1) is close to the students' classroom experience, where mathematics and computing are used in an integrated fashion. Crucially, investigating connections between these domains does not require proof of transfer to a different context: finding evidence of transfer is in itself not a goal of this paper. Nonetheless, the AOT framework's focus on similarities allows for describing the connections students make between domains and their respective representations. In particular, when an activity students engage in shows evidence of being influenced by a previous activity from a different domain, we say that students are making a cross-domain connection.

An example of a study grounded in AOT that explores connections in a mathematical setting is that of Karakok (Karakok, 2019), who looked at physics students' connections among representations of eigenvectors. In this context, Karakok reports on "the necessity of developing flexible shifts between different modes of thinking" and links this to the development of "instructional materials and interventions that emphasize opportunities for students to inquire and connect multiple modes of thinking" [emphasis added]. These are the kinds of connections we are looking for between computing and mathematics.

The modes of thinking Karakok describe correspond to different representations of eigenvectors that each corresponds to a certain point of view about them. These points of view have different affordances for

\footnotetext{
${ }^{1}$ In our case, the actors are students.
} 
the students, for instance, some ways of thinking about eigenvectors are particularly useful when using them in the context of quantum mechanics (Karakok, 2019). The term affordances has often been used in the mathematics education research literature, most notably in the context of technology. The term describes a relationship between an actor and an object that is expressed by some activity: an illustrative example by Gibson (1979) is that water affords drinking and drowning to human beings, while it affords breathing to fish (J. Brown et al., 2004).

Greeno et al. (1993), one of the inspirations for the AOT perspective, discussed affordances in the context of reasoning with representations. This use of the term is notably different from discussing the affordances of computational tools in themselves:

Representations include symbolic expressions that represent actual or potential states of affairs. Representations also include physical constructions such as diagrams, graphs, pictures, and models with properties that are interpreted as corresponding to properties of situations. [...] Affordances for reasoning, on this account, are properties of representations in relation to a person's or group's abilities to use the representations to make inferences. Reasoning is an activity that transforms a representation, and the representation affords that transformational activity. Abilities for reasoning activities include knowing the operations to perform on the notational objects in the representation and understanding the semantic significance of the objects and operations. Conceptual reasoning occurs when representations of concepts are included in the representations that are used in reasoning. [...] Generally, concepts correspond to properties or relations in a domain (pp. 108-109).

Greeno goes on to present algebraic or arithmetic representations as examples that present affordances for reasoning within the domain of mathematics. This paper aims to investigate the affordances of crossdomain reasoning, across the domains of mathematics and computing. To that end, describing the connections (or, in Greeno's terms, the reasonings) that translate representations from one domain to the other is of interest. Such translations are important for making cross-disciplinary connections and have also been considered in the context of transfer (Evans, 1999) and the repair of "communication 'breakdowns' [...] between students, workers and teacher-researchers" (Williams \& Wake, 2007, pp. 345-346).

It should be mentioned that the concept of domains is somewhat contentious and points to bigger questions than we have room for in this paper. For instance, Inagaki \& Hatano (2002) spend some time questioning the conventionally assumed nature of domains in their work on the development of biological knowledge, while diSessa (2017) aims to show that at a fine grain size, domain boundaries appear to be false. While it is certainly worthwhile to investigate and discuss which knowledge belongs to a particular domain, our research questions (see Section 2.3) implicitly assume that the domains of mathematics and computing exist, if only as arbitrary organizing principles. We do not claim that any particular piece of knowledge is inherently mathematical/computational, nor do we suggest that there is no overlap between domains. In fact, if there were no overlap at all, we expect that any connections we identified would be both rare and rather contrived. Our data, as we shall see, suggests otherwise.

In this paper, we will analyze how university students make connections and interpret affordances when working with computer programming to solve problems that integrate elements of mathematics. Henceforth, when we use the term "computing", we will follow Lockwood and Mørken (2021), who consider machine-based computing, i.e., "the practice of developing and precisely articulating algorithms that may be run on a machine". Applied to our context, this means that the students use Python to solve problems that are both mathematical and computational.

The computational representations that our students encountered were mainly of two types: the source code they were working with and the various outputs that code produced when the students ran their programs. We do not claim that these are the only possible representations or activities within machine- 
based computing (and certainly not within computing in general), but our data and interest in classifying connections suggest that this distinction is useful for our purposes. It is entirely possible that different, perhaps finer, distinctions are useful in other contexts.

\subsection{Relevant Literature}

Having established our theoretical perspective, we will proceed to frame this study within existing literature in mathematics education research. More precisely, our study explores connections between mathematics and computing at the undergraduate level. Such studies most often explore a particular subdomain of mathematics, for example combinatorics (Lockwood \& De Chenne, 2020) or statistics (Ramler \& Chapman, 2011). Our mathematical context in this paper spans several aspects of algebra and calculus such domains, namely Taylor series, logarithms, and integration. There is a common theme, however: the computational representation of real-valued variables and functions.

The perceived value of computing in mathematics dates back to the work of Papert (1993), who proposed that the computer offered unique affordances for students learning mathematics. Using the Logo programming language designed for this purpose, Papert studied children's discovery of algorithmic ways of thinking about geometry though use of the computer. The classic example of this is children writing programs that move a Turtle across the screen, tracing out geometrical shapes in the process.

A more recent example that uses the AOT framework in the context of computing is Lockwood \& De Chenne (2020), which explores how students use Python to list and count the outcomes of combinatorial counting problems. One way to achieve this is using conditional statements to eliminate outcomes that do not respect restrictions on ordering, repetition, or both. Lockwood and De Chenne found that this approach focused students' attention on the outcomes they were counting, which again reinforced the conceptual differences between different types of counting problems.

There are also examples of studies that use other frameworks or that cover several mathematical subdomains; Buteau et al. (2016) is an example of both. In this paper, the authors present a case study of a single student spanning three semesters and the student's work on 14 assignments over these semesters. These assignments are connected to many different mathematical topics, and the authors focus on her learning experience across these semesters. This study differs from ours in that they investigate courses designed from the ground up to provide these experiences - our tutorials, on the other hand, are designed to complement a pre-existing course (see Section 3.1 for details). In the context of these courses, the authors describe development processes of exploratory objects that students use to investigate situations and concepts (Buteau \& Muller, 2010).

Going beyond the undergraduate context, there are numerous examples of studies exploring the interplay between mathematics and computing for younger students. Lavy (2006) used Logo to investigate the different types of mathematical arguments such students construct when working in a computerized environment. More recently, Benton et al. $(2017,2018)$ designed interventions using Scratch to have students reason with geometrical concepts and place value. DeJarnette (2019) also used Scratch to investigate the difficulties that schoolchildren face when trying to understand the meaning of symbols and how they fit together. Kaufmann and Stenseth (2020) had young students program in Processing to solve mathematical problems and found that their mathematical arguments became more elaborate over time, but that the trial-and-error method the students often used might counteract this effect to some extent.

This paper aims to provide several examples of how students use computing and mathematics together. These examples are mainly related to the representation of real numbers in computers, and we view them through the lens of student connections and generalizing activity that we presented in Section 2.1. To this end, we will present a framework in Section 4 that allows us to describe the different ways in which students make connections across the domains of mathematics and computing. 


\subsection{Research Questions}

The research questions of this paper are as follows:

1. What types of cross-domain connections do students form between mathematics and code/output?

2. What do these connections afford the students?

3. Which patterns of connections emerge in the students' work?

Research Question 1 will be the focus of the theoretical framework in Section 4, which was constructed based on the analysis of the data. The cross-domain connections also emerge and are exemplified in Section 5, where we apply the framework to examples from the interview data and address Research Question 2. Research Question 3 is addressed in Section 5.4, where we summarize the interviews based on the answers of the first two questions. The patterns we found are shown in the beginning of each Case, however, so the reader will know where we are headed before the evidence is presented.

We emphasize that this paper aims to provide examples of types of connections and their affordances for student reasoning. This paper is about how students work with computing in mathematics - describing the particulars of what the students learned from the tutorials is not our goal here, but we will be addressing this for Case B this in a forthcoming paper (Sand et al., 2021).

\section{Methods}

This study is a qualitative, exploratory case study, applying thematic analysis to identify themes that emerge in a classroom-like interview setting. The goal of this approach is to provide examples of how students connect mathematics and computing, which the stated research questions reflect. We do not aim to investigate what happens spontaneously but have designed tutorials to get students to reason across domains as described in the previous section. The limitations of this approach are discussed in Section 6.1. Its strengths are that it is appropriate for investigating phenomena where prior research is limited and that it focuses on the students' perspectives as opposed to those of experts.

\subsection{Data Collection}

We collected data in two phases: a first phase in the fall of 2019 and a second phase in the fall of 2020, as shown in Figure 1. In both semesters, we recruited students from the course MAT-INF1100: Modelling and Computations, a compulsory first-semester course for students in mathematics, physics, and electronics at the University of Oslo, Norway. This course integrates elements from both computing and mathematics and is taught alongside courses in calculus and programming (Mørken, 2020). As this course appears at the beginning of the students' undergraduate program, it is taught in Norwegian. We conducted the interviews in Norwegian and, later, translated relevant parts to English for joint analysis and publication.

We designed three tutorials that were used both for the interviews and regular group sessions in the course, where students solve and discuss problems. In these group sessions, the students are encouraged to work in groups, and classroom observations confirmed that the overwhelming majority of them do. The task designs are not unimportant or unrelated to our research questions, but we do not have room for a detailed treatment of them in this paper. For that reason, we briefly summarize each of these tutorials in the introduction to the corresponding case in Section 5 and refer to (Sand et al., 2021) for a more thorough discussion on their development. The final versions of all three tutorials are published in (Sand, 2021). 


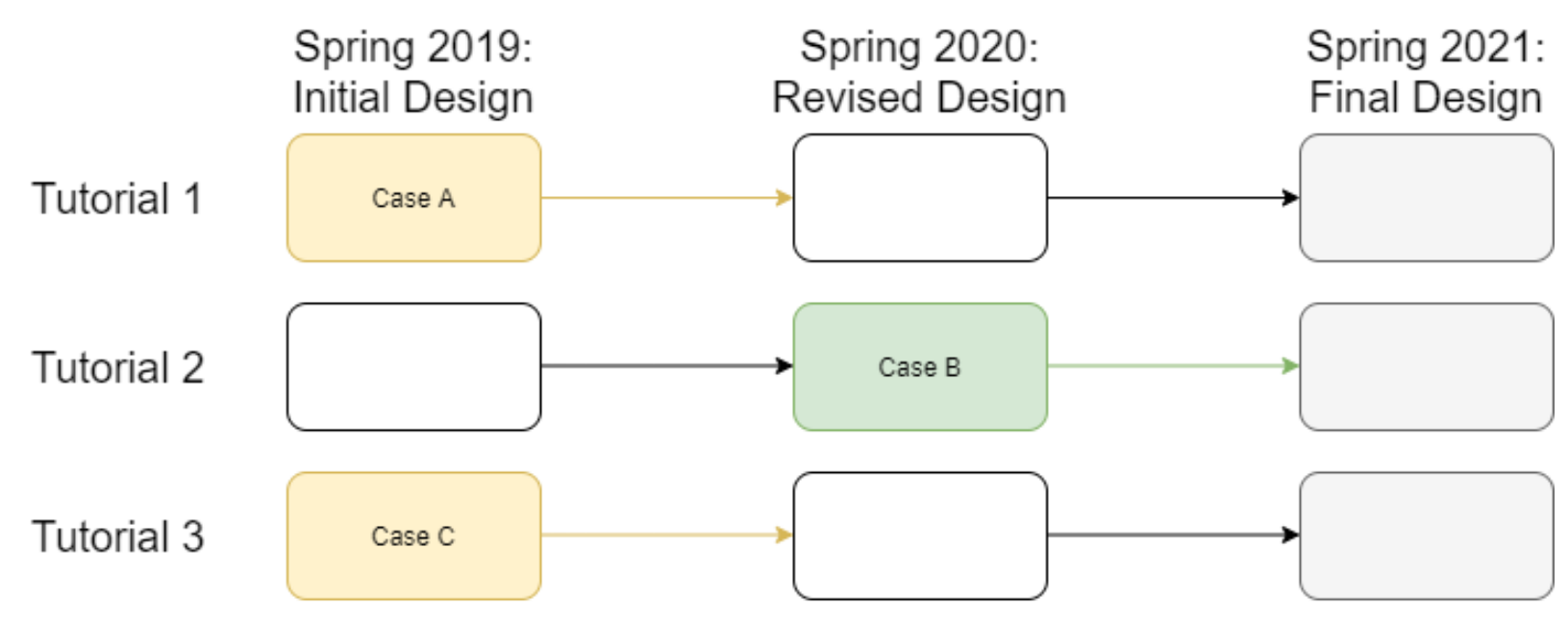

\section{Fall 2019: Initial Interviews \\ Fall 2020: Final Interviews}

Figure 1: Overview of design and data collection process, with the cases in Section 5 highlighted.

The interviews lasted from one to two hours, and we planned to interview groups of two to four students to mirror the way we observed students working in class. The first author conducted all the interviews in person using both video and screen recording on a computer where a familiar Python programming environment had been set up for the students to use. They also had access to a whiteboard, which the video camera captured. The students were told to work together as they would in a normal group session in the course, using the interviewer as a Teaching Assistant (TA) as necessary.

The authors collectively decided on follow-up questions to probe how students perceived their use of mathematics and computing in an integrated way, as well as map their previous programming experience, if any. To avoid these questions having an impact on students' reasoning while they worked on the tutorial tasks, we saved these questions for the end of each interview.

We used classroom observations to recruit participants by inviting groups of students that already worked together. This introduces a bias in our selection beyond that of recruiting volunteers, as we tended to invite students who were adept at verbalizing their thought processes to maximize the quality of our data. However, as the study is merely looking for interesting examples of connections, not any systematic and inclusive analysis, we find this bias acceptable. Nonetheless, we emphasize that the students we interviewed in the first round may not be entirely representative of the student population as a whole, as they were handpicked by us.

In the second phase of interviews (fall of 2020), we kept using SMS as the communication channel, but did not perform classroom observations due to COVID-19 concerns. To ensure no mixing of cohorts, we returned to using online forms in a lecture and asked students to indicate which group session they belonged to and which students they usually worked with. Thus, while we still got students who knew each other from class, in the second round they were not handpicked in the same way.

Some of the interviews in this phase had to be performed digitally. We did not end up using data from these digital interviews, however, as we found this change in format to affect the quality of the data. The interview protocol remained largely unchanged in the second phase.

In total over both phases, we interviewed 20 different students in 15 interviews. Some groups of students were invited back for a second or third interview (only one group declined, again due to COVID concerns) whenever we found that they gave us interesting data. This may have contributed to a bias toward what we 
found interesting in both rounds. We found the possibility that our results may be more the exception than the norm to be an acceptable risk since one of our goals is to provide examples of students connecting mathematics and computing: determining the degree to which our results are representative of the student body at large is deferred to follow-up studies with more appropriate methodology for that purpose.

\subsection{Analysis}

Once we had the data in hand, we performed a thematic analysis with the initial goal of identifying how students integrated mathematics and computing in their work. Among the strengths of thematic analysis are its usefulness for "examining the perspectives of research participants, highlighting similarities and differences, and generating unanticipated insights" (Nowell et al., 2017, p. 2). Its limitations are that its flexibility can lead to incoherence and there are limits to the kinds of claims one can make compared to other theories of qualitative research. Nonetheless, we found that such an approach served as a good first step into unfamiliar territory, and that it followed the six-page approach outlined by Nowell et al. (2017).

3.2.1 Familiarizing Yourself with Your Data. At the end of each semester, we had all the audio from the interviews transcribed. The first author familiarized himself with the data and reviewed the transcripts to divide them into short segments with brief descriptions of each. Segments were flagged for further review if they (a) involved both mathematics and computing, and (b) the students' work was focused on making sense of something, and these were then shared with the co-authors for further discussion.

In the process the authors found several episodes (usually consisting of several consecutive segments each) where students either (a) were engaged in some activity that contained elements of both computing and mathematics, (b) made a transition between computing and mathematics activities, or (c), both. Our interest in these connections influenced our choice of AOT as a lens through which to understand the data. Initially, we made a broad pass and subsequently selected the most promising episodes, focusing on justifying why these episodes should be a priority. Cases A and C in Section 5 came out of this pruning process.

The first author then translated the transcripts from Norwegian into English and enhanced them with evidence from other sources, such as screen and video captures, photos of the whiteboards and collected worksheets. These enhanced transcripts were shared with the co-authors and formed the basis of the analysis from that point onward.

3.2.2 Generating Initial Codes. Initially, the first author divided each episode into short segments and coded each segment. We then looked at the bigger picture, focusing on the patterns that were apparent from the students' point of view. Based on this, the first author formulated claims about the students' process of making sense of mathematics using computing, supported by evidence from the transcripts, in analytical memos. The co-authors reviewed the claims and their justifications for the sake of validation and helped refine the claims in several steps. Examples of these initial codes were "pure coding", "pure math", and "integrated", reflecting our interest in the integration of computing in mathematics. As a starting point, we assigned confidence levels to these labels, and the first author then grouped the high-confidence segments together to see what they had in common. This work became the foundation for our analytical framework for labelling connection types.

3.2.3 Searching for Themes. We then assigned labels to the episodes, identifying and highlighting key actions that we took as justifications for this coding. Examples of these include verbal utterances from the students, their written work in the code editor or the whiteboard, and other actions caught on tape. These key actions are listed in the "Evidence" column of Table 1 of Section 4. 
We assigned confidence levels to these labels, and the first author then grouped the high-confidence segments together to see what they had in common. This work became the foundation for our analytical framework for labelling connection types (Section 4).

In the process, detailed notes were kept of each version of these descriptive labels and their development was triangulated among the co-authors. Examples of early themes or labels were "mapping code to mathematics", "mapping mathematics to code", and "explicitly telling the code what to do". These correspond to early versions of the connections in Figure 2. Realizing that we had to define these arrows/labels more clearly, the first author suggested names and descriptions for each, and the other authors contributed to the discussions about how to define these. During these iterative discussions, the three properties of Medium, Structure, and Syntax emerged, as shown in Table 1 (Section 4).

3.2.4 Reviewing Themes. After several iterations, we decided to focus on cross-domain connections alone, ignoring connections that belonged exclusively to one of the domains (all mathematics or all computing). We created new enhanced transcripts with a separate column for coding and key actions to get a clearer picture of the codes as applied to our raw data, including data we had not previously analyzed in detail. The triangulation and discussion from Section 3.2.3 continued throughout this phase.

3.2.5 Defining and Naming Themes. Finally, we also linked the key actions to connections or noticing similarities. In the AOT literature we found nothing about connections between domains in general, even though (Lockwood \& De Chenne, 2020) is another example of the same. In this stage, we named the connection labels in Table 2 (Section 4) and described each of them.

We also summarized what each of the selected episodes demonstrated and refined our coding of these based on this. The process described in Figure 4 (Section 5.1) was identified at this time, at which point we also identified the half-connections described in footnote 4 of Section 4 (see also Case A and B in Section $5)$.

3.2.6 Writing Up the Report. Finally, we went back into the data and re-coded them using the updated labels and properties in Section 4. At this point, we also had available transcripts from the second phase and highlighted episodes from this second round of data, where we recognized that the emerging framework could be used for analysis. Case B in Section 5.2 was identified at this stage, as well as a new label (Output Modelling) that had not emerged in the first phase data.

We also sent an advanced draft of this paper to all the students in Cases A, B, and C to ensure that we did not misrepresent them or accidentally allow them to be identified from the interview excerpts.

\section{Analytical Framework}

In this project, our focus is on student connections that straddle the domain boundary between computing and mathematics. We would like to note that it is not completely trivial to identify what is a mathematical activity, what is a computational one, and what is both. Additionally, while our focus is on connections made from a student perspective (Section 2.1), we rely on the expert perspective for distinguishing between domains. This section explains how we do that.

As mentioned in Section 2.1, we distinguish between two subdomains of computing: Code (programs the students write in the code editor) and Output (terminal window printouts and plots produced by these programs), as we observed that students tend to interact differently with each of these aspects. We take the Code as a mutable set of instructions for the computer to interpret literally, whereas the Output is a result of the computer's activity. The students can only affect it indirectly, by altering the Code or input to the program. 
After categorizing relevant segments in our data, we developed a classification scheme to label these connections, and these labels became a useful lens for the analysis. There are many ways to classify what is mathematical and computational, and we acknowledge this without claiming to be authorities on the subject. In this section, we are merely describing how and why we consider something to belong to either domain, as a result of the process described in Section 3.2. These criteria are summarized in in Table 1.

Table 1. Properties used for labelling connections.

\begin{tabular}{|c|c|c|}
\hline Property & Meaning & Evidence \\
\hline Medium & $\begin{array}{l}\text { The physical/visual medium students work with. In our } \\
\text { use of the terms, } \\
\text { Math: paper, whiteboard (physical or digital) } \\
\text { Code: a code editor } \\
\text { Output: plots or terminal windows }\end{array}$ & $\begin{array}{l}\text { Observing actions } \\
\text { (Verbal utterances) }\end{array}$ \\
\hline Structure & $\begin{array}{l}\text { The ways in which students interpret or organize } \\
\text { information in the Medium } \\
\text { Math: mathematical arguments (such as equations) } \\
\text { Code: stepwise instructions, such as Python code or } \\
\text { pseudocode } \\
\text { Output: real or imagined output produced by a program } \\
\text { (for instance plots or tabulated numbers) }\end{array}$ & $\begin{array}{l}\text { Verbal utterances } \\
\text { Students' written work }\end{array}$ \\
\hline Syntax & $\begin{array}{l}\text { The ways in which students represent individual pieces } \\
\text { of information } \\
\text { Math: no concern with storage of values or data types, } \\
\text { short variable and function names } \\
\text { Code/Output: typed values, focus on storing them in } \\
\text { memory, descriptive variable and function names }\end{array}$ & $\begin{array}{l}\text { Verbal utterances } \\
\text { Students' written work }\end{array}$ \\
\hline
\end{tabular}

To assign a label to a connection, we examined it along the three dimensions or properties of Medium, Syntax, and Structure. Some of these properties mirror the discussion of symbolic notation found in DeJarnette, (2019). In particular, Syntax and Structure are reminiscent of "vocabulary" and "grammar", respectively: the former describes the meaning of specific symbols, whereas the latter refers to the way the symbols are connected to communicate meaning.

For example, to infer the Syntax, if suffices to investigate how students represent and talk about a particular symbol (such as a variable), but one needs to take into account many symbols and their relations to one another in order to classify the Structure. Our use of Structure is also reminiscent of Sherin's (2001) discussion of structures in symbolic forms in algebraic and programming formulations of physics, respectively. The question of translating between these formulations then presumably requires the kind of connections that we investigate in this paper.

For Syntax, we are making the assumption that students do not use notation systems at random, but use them to indicate and communicate which domain they are working in. Note that on occasion, this usage may be ambiguous and point to more than one domain. In these cases, supporting evidence is needed to classify the connection. 
For a particular connection, each of the three properties in Table 1 can be valued Code, Output (both subdomains of computing), or Math. Importantly, we use them to identify two domains: the Source Domain relates to the past activity students are referring to, while the Target Domain applies to the current activity taking place as the connection is made. Simply put, when we sort the activities chronologically, the past activity becomes the Source and the current one becomes the Target.

We will exemplify these ideas further in Section 5, but here is an illustrative sample: Two of our students, Gina and Benjamin, had constructed a proof on the whiteboard (See Figures 5 through 11 in Section 5.1) that the code they were working on should produce the same output regardless of what the input was. Benjamin then looked at the program's output on the computer screen (Figure 12), asking why the program's actual output did not correspond to the theoretical result.

In this instance, we identified the Source Domain as Math. Preceding this move, the students had worked on the whiteboard (Medium), describing their work as solving an equation (Structure), using mathematical conventions for variable names and untyped numbers ${ }^{2}$ (Syntax). Then, Benjamin focused his attention on the terminal window containing the output (Medium), where numbers had been printed in a tabular format (Structure) as finite-precision computer numbers (Syntax). We therefore identified the Target Domain as Output. Note that connections need not always be made to the activity immediately preceding the connection: sometimes students would refer to ideas brought up in lectures, for example.

A cross-domain connection, such as the one in the example above, is recognized by the Source and Target Domains being different: not both belonging to mathematics or computing. There are four types or labels that we have assigned to the connections we found in our data, see Table 2 . We acknowledge that this need not be an exhaustive list, but it serves as an account of the kinds of cross-domain connections that we observed in our interview data. These connections strongly resemble reasoning as described by Greeno et al., (1993, p. 109): "an activity that transforms a representation, and the representation affords that transformational activity."

Table 2: Connection labels.

\begin{tabular}{|c|l|l|}
\hline $\begin{array}{c}\text { Label } \\
\text { Math }\end{array}$ & Source Domain & Target Domain \\
\hline $\begin{array}{c}\text { Code } \\
\text { Modelling }\end{array}$ & $\begin{array}{l}\text { Computing } \\
\text { (Code) }\end{array}$ \\
\hline Output Modelling & $\begin{array}{l}\text { Code) } \\
\text { Computing } \\
\text { (Output) }\end{array}$ & Math \\
\hline $\begin{array}{c}\text { Mathematical } \\
\text { Interpretation }\end{array}$ & Math & $\begin{array}{l}\text { Computing } \\
\text { (Output) }\end{array}$ \\
\hline
\end{tabular}

We label a connection (1) Math Implementation when the Source Domain is Math, and the Target Domain is Computing (Code), hereafter shortened to simply "Code". An example of Math Implementation is when the students write a loop in code to calculate the sum of a series of numbers that follows a particular pattern.

\footnotetext{
${ }^{2}$ In computing, the constants 1 and 1.0 are different because they have different data types. The former is an integer, while the latter is a finiteprecision floating-point number, and these are treated differently by the computer. Both, of course, represent the same mathematical value of one, and when the students interpret the numbers mathematically, they make no distinctions between the different data types that can represent the mathematical value.
} 
We label a connection (2) Code Modelling when the Source Domain is Code, and the Target Domain is Math. An example of Code Modelling is when students produce a mathematical proof of concept for a piece of a program that they are designing or that that someone else has written.

We use the label (3) Output Modelling when the Source Domain is Output, and the Target Domain is Math. An example of Output Modelling is when students make use of mathematics to determine the required changes to a program that makes it produce some desired output (instead of resorting to trial and error). Where Code Modelling is mainly associated with designing or attempting to understand programs, Output Modelling is instead associated with tweaking or refining a program that is already implemented and reasonably well understood, such that the students are mainly preoccupied with the input and output and not the transformation of one into the other.

Finally, we have (4) Mathematical Interpretation, meaning that the Source Domain is Math, and the Target Domain is Output. An example of Mathematical Interpretation is when students call on mathematical knowledge as they are engaging with the output of a program. For instance, they could be verifying that the Output makes sense mathematically or making informed decisions on how to improve the program going forward. Unlike Output Modelling, they are attending to plots, tables or files produced by the program instead of the Math itself.

Note that while Table 2 might suggest the Source and Target Domains need to be classified with certainty, we did allow for exceptions. In some cases, the three properties of Table 1 instead painted a hybrid picture, where Medium, Structure and Syntax did not all point in the same direction. On two occasions, we came to interpret these hybrids as half-formed connections that the students later returned to and then connected fully. While these "half-connections" are not our primary focus in this paper, they are still relevant, as a pair of them can be interpreted as a full connection happening in two distinct stages. As an example, consider the stages of a connection that we will examine more closely shortly, in Case A:

1) Source Domain: Code $\rightarrow$ Target Domain: Math/Code hybrid

2) Source Domain: Math/Code hybrid $\rightarrow$ Target Domain: Math

In Figure 2, we illustrate the labels from Table 2 with arrows originating from the Source Domain and pointing toward the Target Domain. In short, the arrows point from the past activity to the present. As discussed in Section 2.1, the four types of connections represent the similarities that the students (the actors) see between activities.

\section{Results}

This section contains our three cases, one from each tutorial. We will describe the students at the beginning of each case, after we provide a summary of each tutorial as necessary background. Cases A and C are from the first interview phase (fall 2019), while Case B is from the second (fall 2020).

These cases demonstrate four different ways that students integrate computing and mathematics, that we summarize in Section 5.4. Each of these patterns represents a way that students chained connections together. This, along with the properties in Table 1 and the connection labels in Table 2, add a third level of granularity to our classification of the data. On the most detailed level (properties), we examined the Medium, Structure, and Syntax to determine which connection labels to assign. Then, we labelled individual connections. Finally, we identified patterns of connections that occurred dependently on one another.

This progression from a fine grain size to the bigger picture is to some extent represented in our presentation of the analysis. In Case A, we are more explicit about Structure and Syntax in order to provide examples of how the properties of Section 4 were used in the analysis. In Cases B and C, on the other hand, this is assumed sufficiently familiar to the reader that the focus in these later subsections shifts to the 
connections in Figure 2 and the patterns they form. Medium is very often evident from the data directly but is still part of the analysis despite rarely being discussed at length.

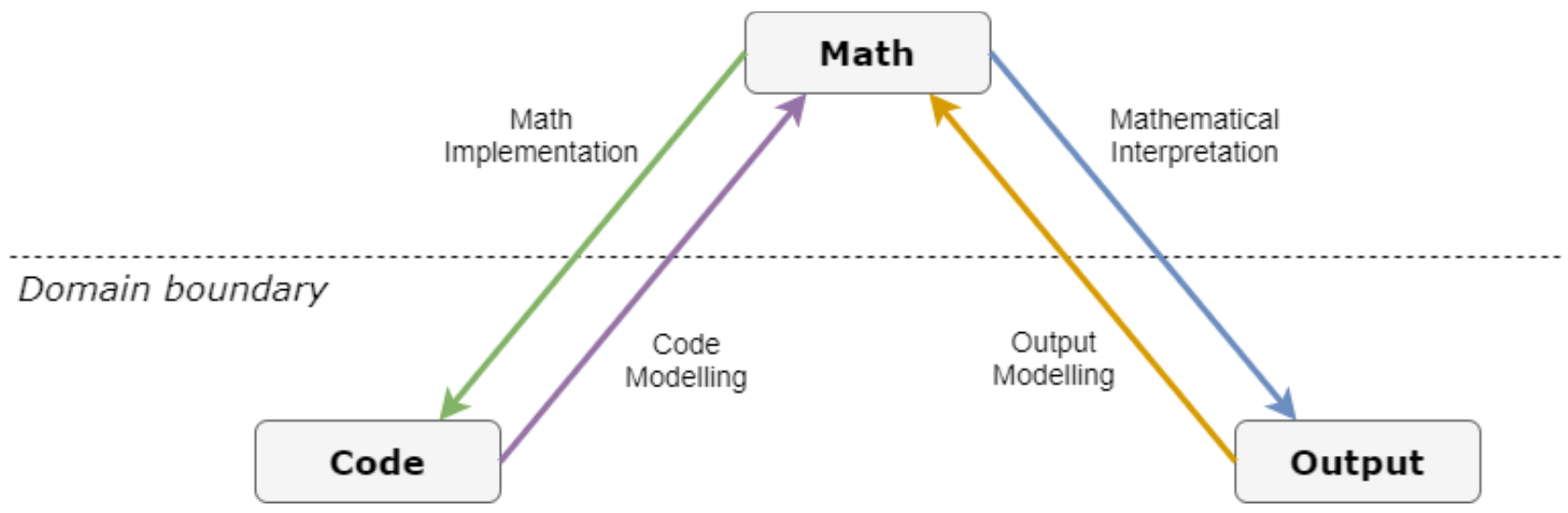

Figure 2: Cross-domain connection labels, corresponding to the rows in Table 2. Note that the name of the label always corresponds to the starting point of the arrow, which represents the Source Domain.

\subsection{Case A: Gina and Benjamin (Tutorial 1, 2019)}

In our first case, we focus on two students, Gina and Benjamin. This case highlights the ways in which these students used mathematics on a whiteboard to model a piece of code. In the process, they constructed a proof of the code's correctness. At that point, the students' focus shifted to the output produced by the program, where they sought to explain it based on their mathematical considerations. This, using the framework of Section 3.2, constitutes an example of Code Modelling followed by Mathematical Interpretation.

5.1.1 Tutorial 1: Rounding Errors. Before we present excerpts of the interview followed by our analysis, we provide a summary of the tutorial as necessary background, as we will do for all the cases under consideration. In the first tutorial, the students were presented with a short program designed to take a randomly generated number between 0 and 1 and then calculate what number (the step size) must be added to the original random number 9 times to get exactly 1.0 as the result (see Figure 3).

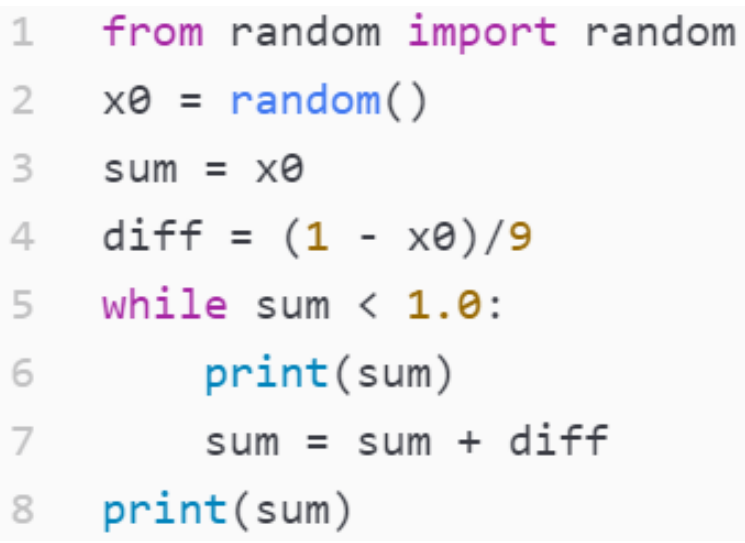

Figure 3: The program from Tutorial 1, written by the first author. 
In practice, however, the program did not produce 1.0 every time, even though the code contained a while loop that kept running as long as the sum up to that point was less than one. The point of this task was to raise the issue of rounding errors, as a rounding error in the sum would frequently cause the loop to continue for an additional iteration beyond what was intended, leading to a large error in the final answer.

We tasked the students with testing the code and finding out what, if anything, was wrong with it. At the end of the tutorial, they were given a set of exercises to help them understand how the computer's binary representation of real numbers determine where rounding errors will appear and where they will not.

5.1.2 Overview of Case A. We saw Gina and Benjamin use mathematics to model a program that we gave them, in order to grasp how it was supposed to work. After having translated the program to mathematical symbol language, they were able to take those expressions and treat them like mathematical quantities such as equations. We posit that this kind of algebraic manipulation would not have been so easy for them to do in a strictly computational setting, as it would require knowledge of sympy or similar Python libraries.

Gina and Benjamin followed up this work by going into Mathematical Interpretation of the Output, allowing them to link Code and Output together through Math instead of using the program as a black box to produce the output for them. We have named this pattern of connections Replicating Program and depicted it in Figure 4. Note that this only characterizes these students in part of the interview we used for Case A - in other parts of the interview the same students' connections may be described differently (the same applies to Case $\mathrm{B}$ and Case $\mathrm{C}$, as we shall see later).

For readability, the whiteboard figures in Case A are accurate reproductions by the first author of the students' written work as captured on video.

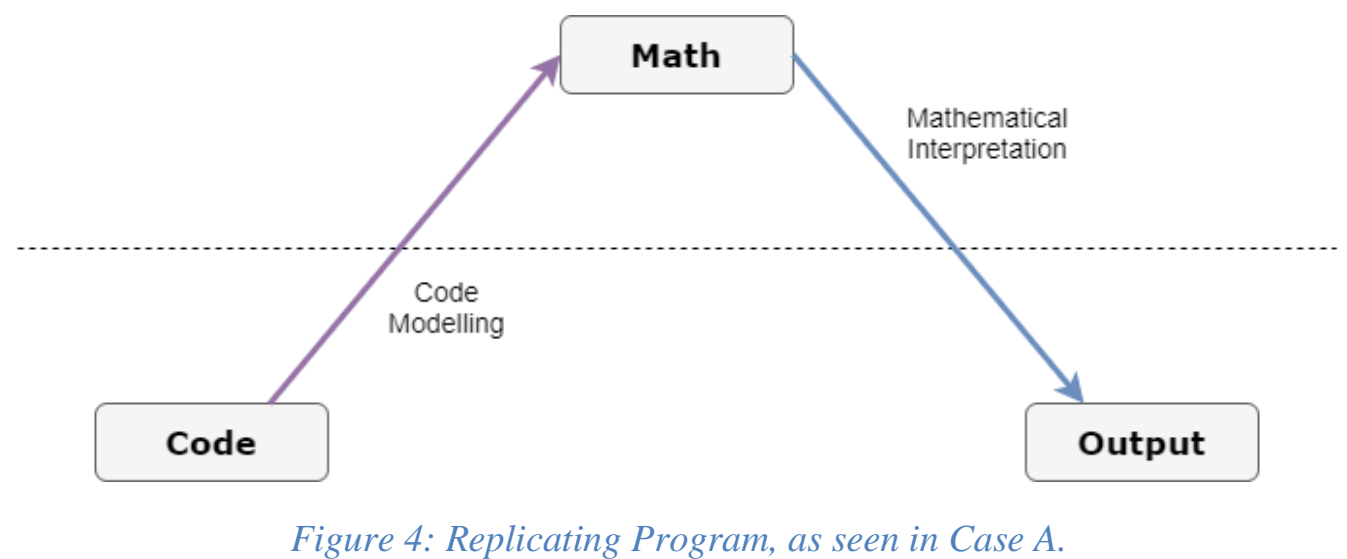

Gina and Benjamin were first-year students, neither of which had any previous experience with programming prior to the semester. By the time of the first interview, they had two and a half weeks of Python experience. Gina self-identified as a "problem solver" and tended to verbalize her thinking, including working theories and understandings. Benjamin tended to speak somewhat less than Gina and would typically comment on the work Gina did on the computer or whiteboard. At times, Benjamin would take over the computer or whiteboard and direct the conversation.

5.1.3 Code Modelling. Gina and Benjamin had spent some time discussing what the code did, and they ran the program a few times. Subsequently, they used the whiteboard to mathematically describe an imagined case where the random number was 0.55 , and the resulting step size 0.05 . They predicted what the program would print in such a case, and Gina noted explicitly that she recognized this as uniform spacing. 
The students then focused on the code for a while, trying to identify errors that had little to do with the mathematics, such as using the "wrong" initial number. They started discussing what the piece of code was supposed to accomplish, but soon began to express some hesitation and doubt.

At this point, Gina turned to the whiteboard and wrote the expression in Figure 5, presumably to help move the process along. While writing, she uttered: "9 times, we were sort of supposed to... 1 plus blah blah blah, and up to dif $f_{9}$, eh [...] and all of this together should become 1."

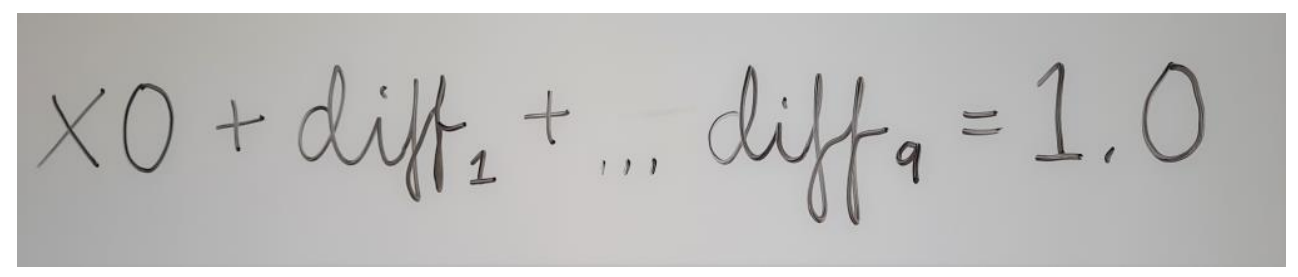

Figure 5: Gina's first expression.

We suggest that this change of activity represents the beginning of a cross-domain connection from Gina and Benjamin's point of view. Gina's expression contains the variable names $x 0$ and diff $_{1}$ that we recognize from the code in Figure 3, and her verbal description of what she is doing makes it clear that she constructs a connection between the previous activity of attempting to understand the code and what she is writing.

We have labelled this connection as an instance of Code Modelling (see Table 2). Interestingly, this connection happens in two stages, where the exchange above marks the first one. A little later in the interview, we will see the students make a further connection from this work to something they regard as fully mathematical, completing the Code Modelling. But first, we present our justifications for the labelling applied here.

The Source Domain (the idea being represented) is Code because her expression models the mathematical operation that the code is performing on the left-hand side and the desired outcome on the right. This points back to their engagement with the code in the code editor just moments prior. We identify the Target Medium as Math from Gina's writing on the whiteboard in Figure 5. Structure and Syntax are more difficult to pinpoint in this instance.

While the Target Structure in Figure 5 certainly looks like Math to an observer, we shall see that both Gina and Benjamin displayed excitement later, when they realized that this statement could in fact be interpreted as an equation. It is therefore problematic to claim that the Target Structure was Math from the students' point of view before they made that realization.

The Target Syntax is an interesting hybrid between Code and Math, containing some elements of each. We recognize Code syntax in the variable names, as $x 0$ and diff 1 are translated directly from the code in Figure 3 and not converted to traditional mathematical syntax like for instance $x_{0}$ and $d_{1}$. Furthermore, in mathematics it would be considered unnecessary to write the constant 1 as 1.0 , whereas in computing, 1 and 1.0 are values with different data types (integer and floating-point number, respectively). Gina said 1 aloud but wrote 1.0, and we infer that this means she connects the computationally typed value (1.0) to the mathematical number (1). It is possible, however, that these numbers were also translated directly, just like the variable names.

Math Syntax was also present: Gina wrote the while loop as a sum (because the loop adds one term each iteration), using subscripts 1 and 9 to label the different iterations. The use of "..." on the whiteboard to mark repetition is a conventional mathematical way of describing the sort of repetition that the loop represents, and her saying "blah blah blah" as she wrote it implies that she meant it in that way. 
We interpret the equality in Figure 5 to represent either a mathematical assertion or a computational evaluation from the students' point of view ${ }^{3}$, because throughout the interview the students demonstrated that they knew that in Python, variable assignments require a single variable on the left-hand side to store the value of the expression on the right. This hybrid syntax laid the groundwork for their subsequent interpretation of Gina's expression as something mathematical.

In sum, we interpret this event as the students writing a pseudo-mathematical expression on the whiteboard that they regarded as similar to the code. We find it interesting that they did so on their own accord, without prompting.

Gina went on to say:

Gina Okay, you can surely write a, that is, by hand we can surely write something sum of [inaudible].

Benjamin Yes, that is, because all those diffs are equal then. [...]

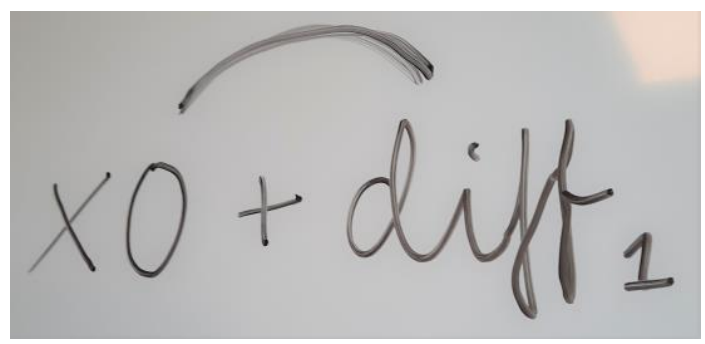

Figure 6: Line connecting $x 0$ and dif $f_{1}$

Gina So it is, but the diffs are in a way dependent [connects $x 0$ and diff $f_{1}$ with a curved line as seen in Figure 6], that is, these are connected because the expression for diff is 1 minus $x 0$ [writes the statement in Figure 7].

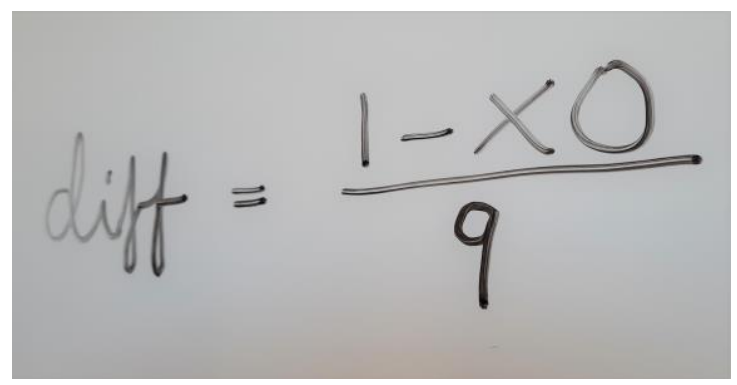

Figure 7: Gina's second expression.

Benjamin Yes, but that happens outside the loop. [...] So, it is just calculated once.

Gina's choice of words "by hand" emphasizes that she sees the Target Medium as Math-related. Her mention of writing something as a "sum" could indicate that she does not regard the Target Structure as

\footnotetext{
${ }^{3}$ From an expert perspective, the equality symbol = may take on different meanings when applied to real numbers, as illustrated by the following example that we borrowed from Brown (2017): Consider the statement $x=x^{2}$, which might be (a) an assertion constraining the value of $x$ to members of the set $\{0,1\}$, (b) an assignment where the value on the right-hand side is computed and then assigned to the variable, so that if $x$ previously had the value 8, the new value becomes 64, and (c) an evaluation of the expressions $x$ and $x^{2}$ that returns True if their values are identical and False otherwise. While (a) limits the value $x$ to 0,1 , or both, (b) causes $x$ to become a non-negative number (which one it becomes depends on its previous value), and (c) neither constrains nor changes the value of $x$ at all. Programming languages commonly use a separate operator from $=$ to distinguish between the latter two, such as : = in Pascal (b) or $==$ in Python (c).
} 
being Code at this point. The Source Domain could still be Code, however, if she was thinking of the loop being represented by a sum, which is something the students were likely to have seen in the lecture notes by this point ${ }^{4}$. Gina and Benjamin's explicit use of the word "loop", and their concern with how many times something is calculated both seem to support this interpretation. We therefore infer that in their whiteboard inscriptions, they are attempting to represent Code.

Furthermore, in writing her second expression (Figure 7), Gina indicated explicitly that from her point of view there was a similarity between the variable assignments in the code (the Source Structure) and the mathematical concept of dependent variables (the Target Structure). Because the expression for the value assigned to the variable diff (line 4 in Figure 3) contains the value of $x 0$, Gina realized that these variables were not independent from a mathematical point of view. Furthermore, she refers to the variable assignment of diff as an "expression". This seems to suggest that Gina implicitly believed the mathematical definition to be equivalent to the computational assignment, even if she kept using the variable names directly from the code. The tutorial worksheet text also used the variable name $x 0$ to describe this number, and Gina may simply have followed this naming convention.

At this point, the interviewer intervened, as the students seemed increasingly hesitant and uncertain about what to do next. The interviewer pointed their attention to the nature of their activity and suggested that it might be taken further in the direction of Math:

Interviewer For if you're not just interpreting the task then you're doing something mathematical, and that is...

Gina Yes, clearly, I thought it was hard to understand the task, but maybe that's because there are so many steps here.

The interviewer followed up on this by asking Gina and Benjamin what they were doing on the whiteboard, why they were doing it and what they would use the result for. Gina responded that she supposed it was to visualize for themselves what the problem was.

We find these last two exchanges to be interesting for two reasons. First, they reveal what the students thought they were engaged in (visualizing does fit with our label of Code Modelling) and why (there were many steps to the task, and it was hard for them to understand). Second, it is possible that the students' familiarity with mathematical syntax as opposed to Python syntax could factor into their decision to model the code's behavior in this way.

At this point, Benjamin seemed to pick up on the interviewer's suggestion that the work on the whiteboard could be interpreted as something more mathematical. Note that we are not limiting our investigation to what students connect spontaneously. Even though the interviewer is pushing the students in a particular direction here, the students may still react to this in a number of ways, for instance ignoring the interviewer's statement. We are also interested in how instructors can influence the connection-making of students, and in this case name-dropping mathematics was sufficient. While looking at the whiteboard, Benjamin interrupted the conversation between Gina and the interviewer and proposed inserting the expression from Figure 7 into that of Figure 5, resulting in the expression in Figure 8:

\footnotetext{
${ }^{4}$ See Subsection 1.4.3 in (Mørken, 2017), where two simple sums are represented by loops in one of the first introductions to algorithms. It is possible that this presentation could have made the students think of sums and loops as equivalent in some cases.
} 


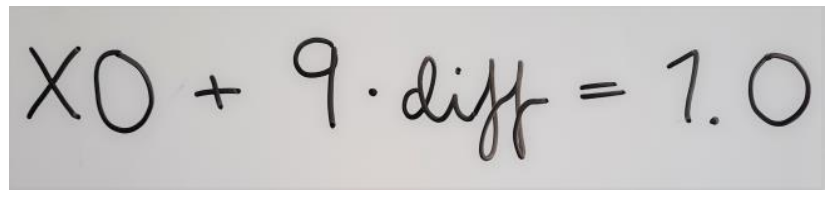

Figure 8: Benjamin's first expression.

Gina Yes, that's an equation!

Benjamin That's an equation, so if we now insert that one [indicates Gina's second statement in Figure 7], then that's basically $x 0$ divided by 9.

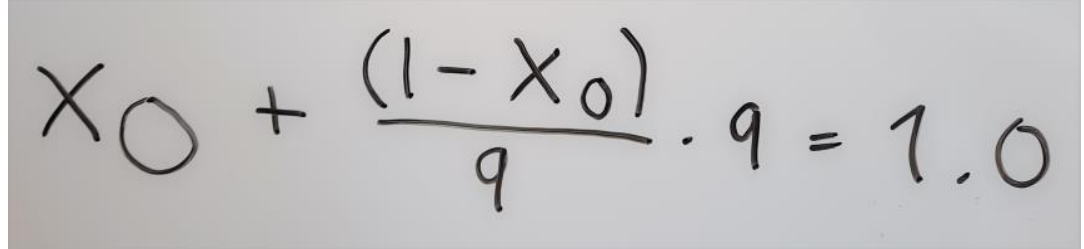

Figure 9: Benjamin's second expression.

\section{Gina [eagerly] Yes!}

We now conjecture that this sequence of statements completes Gina and Benjamin's Code Modelling connection, for three reasons:

First, what Benjamin did here was to perform a mathematical substitution using Gina's two statements. This appears to be an affordance of interpreting their whiteboard model as a mathematical construct, because even though Gina noted the dependence of diff on $x 0$ earlier, she did not substitute diff for its definition at the time. After all, no such substitution was evident in the code, so for the purpose of "visualizing" alone, there was no need to do this. We conjecture that substitution is an affordance of Code Modelling.

Second, we saw Gina and Benjamin's exclamations of excitement during this exchange. These suggest that in the students' eyes, this was a new possibility that had just surfaced, but had not been evident before. This supports our earlier claim that Gina did not think of the Target Structure as being Math until this point.

Finally, we note a change in Benjamin's use of the Target Syntax in response to Gina's equation statement. In his first statement (Figure 8), he continued using Gina's syntax with no subscript in $x 0$. In his second (Figure 9), he adopted the more mathematically conventional $x_{0}$, which he kept using from that point on. The multi-symbol variable $\operatorname{diff}$ had been substituted away, leaving the constant 1.0 as the only relic left over from the earlier mixed syntax that contained both Math and Code elements. We interpret this as a clear shift in the Target Syntax from a hybrid state towards Math in response to the interviewer's suggestion.

Benjamin proceeded to solve the equation analytically. His work on it is shown in Figure 10 and Figure 11. At the end, Gina seemed disappointed in the result: "Yes, wow! It cancelled, okay, eh [...] That was a little disappointing, but it's probably that way I... be a sensible way to set it up, but..."

We conjecture that Benjamin's solution of the equation for $x_{0}$ is another affordance of Code Modelling. The students made no attempts to solve for anything while thinking of what they were doing as "visualizing" the code. It is possible that Benjamin's solution attempt was guided more by the affordances in the Target Structure than by the stated goal of the task: to find out what, if anything, was wrong with the program. 


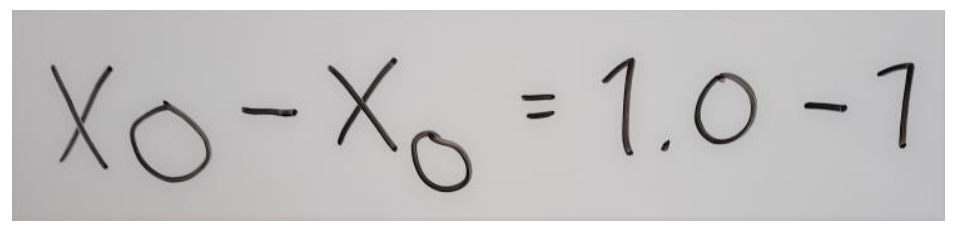

Figure 10: Benjamin's third expression.

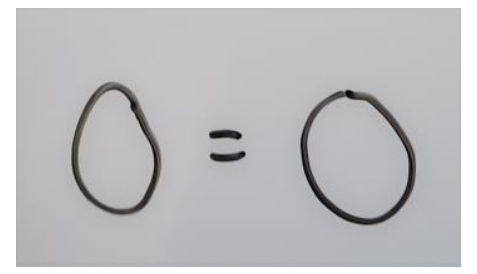

Figure 11: Benjamin's final expression.

As far as Target Syntax is concerned, in Python, 1.0 - 1 in Figure 10 would be a legal operation, but, under the hood, the integer value 1 would be converted to a decimal number. Hence, the computationally typed result of such a calculation would be 0.0 . Benjamin's choice of representing the answer as the integer 0 is further justification for our claim that the Target Syntax is Math at this point, where data types are not considered important. We do not claim that Benjamin consciously left 1.0 as a float value and then made this conversion we described, however. Rather, we believe that Benjamin carried over 1.0 unchanged from Gina's first expression even after the change of Target Syntax to Math (perhaps as an oversight) and then converted it to mathematical syntax as soon as the value changed.

The final $0=0$ expression (Figure 11) is interesting for other reasons. From an observer perspective, one may interpret the result $0=0$ as a proof that the equality holds for all values of $x_{0}$. In an equation, a relationship that always holds, such as $x+x=2 x$, is reducible to $0=0$ through algebraic manipulation. In contrast, a contradictory statement, such as $x=x+1$, would yield $0=1$ through the same means. In both cases, the variable $x$ has been eliminated, showing that the truth or falsity of the relation that the equation represents does not depend on the value of that variable.

However, taking an actor-oriented perspective, we infer that Gina's comments reveals that she was not viewing $0=0$ in this way. Rather, she seemed to be almost disappointed in the result and did not see it as helping them progress toward their goals. We speculate that even though this demonstrates constructing a proof as yet another affordance of Code Modelling from our point of view, Gina did not see it as such. Instead, she might have expected to find a particular value of $x_{0}$ that would have made the program behave as intended. In introductory mathematics courses, students are frequently asked to solve for some variable to find a value that satisfies a constraint represented by the equation, and it is not surprising if Gina interpreted the goal of the activity in this way.

With this interpretation in mind, the equation failing to result in a satisfying value might have suggested to her that either such a value does not exist, or the method itself was inadequate to identify it. We conjecture that Gina did expect this method to produce a result in most instances, due to her stated belief in it being a "sensible way to set it up", but that it failed in this particular case. Hence, for Gina, their strategy seemed like it would be useful in general, just not in this particular instance. 
5.1.4 Mathematical Interpretation. Benjamin appeared to be of a different mind, however, as he used the computer to switch from the code editor to the output in the terminal window shown in Figure 12: "But, why isn't it working here then, why doesn't that become 1?"

We interpret from this remark that Benjamin expected the result in Figure 11 and the output in Figure 12 to be connected, in the sense that the output should have been 1.0 in both and expressed concern that this seemed not to be the case. In the following, we have treated this expectation of similarity as a different connection than the previous one. In fact, this is the connection we used as an example in Section 3.2. We interpret this connection as an example of Mathematical Interpretation.

Benjamin acted as though, from his point of view, the program's method had been proven, although he did not articulate such a belief: If he shared Gina's belief that the program would only work for a certain value of $x_{0}$, or even just that their method was unable to find such a value, we do not think he would act surprised that the program did not produce the expected answer for the two examples in the output. In fact, that would be rather expected behavior from the program. We conclude that Benjamin saw the affordance of constructing a proof that Gina did not. The question for him then became why the program failed to reproduce the predicted output.

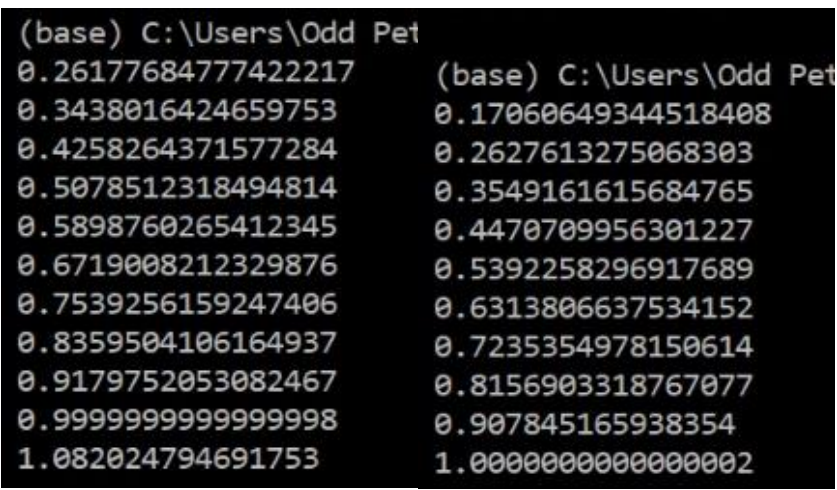

Figure 12: The output visible in the terminal window, showing two rounding errors: one that makes the loop take an extra iteration (left) and one that does not (right).

From an observer point of view, a non-mathematical reason such as rounding errors would explain the result without invalidating the proof. Because the students did not take such errors into account in their whiteboard model, their appearance (in theory recognizable in Figure 12) might have pointed them toward the source of the problem if they had followed this line of reasoning. We do not claim that this insight was available to Benjamin at the time, however. He seemed to initially trust the mathematical proof but then was surprised the code's inability to accurately reproduce the expected result. To the extent that he saw his work as a proof, it is possible that the output made him doubt it.

This might help explain why Gina managed to convince Benjamin that they had to find a particular value for $x_{0}$ that made the program work as intended. While at odds with the stated goal of the task, this belief nonetheless prevented Gina and Benjamin from focusing on this important difference between mathematical theory and computational practice until the interviewer intervened.

5.1.5 Summary of Case A. In this case, we identified that the students made cross-domain connections, and we explored what these connections afforded. First, Gina's uncertainty about how the code worked led her to transition into modelling it using the more familiar (to her) syntax of mathematics. This afforded 
their focus to shift from the code itself (and its output) to what it represented (the mathematical way these results were calculated).

Second, the interviewer's observation that the model of the program could in fact be interpreted as something inherently mathematical seemed to cause Benjamin to ignore what the statements represented and instead take them as mathematical givens. This afforded the use of the students' repertoire of mathematical tools, resulting in a proof of concept for the program. We interpret this as the completion of the connection that began with Gina's first attempt on the whiteboard.

Finally, Benjamin's apparent acceptance of the proof led him to shift his focus to the code's output, to make sense of the dissimilarity between the mathematical and the computational result. This evaluation could potentially have afforded a useful investigation into the differences between the mathematical statements and the code that they modelled if not for Gina's rejection of $0=0$ as a useful result. Had the result been interpreted correctly, Benjamin's focus on the output would have been a natural entry point into such an investigation.

\subsection{Case B: Rita and Lena (Tutorial 2, 2020)}

Our second case is about Rita and Lena's work on Tutorial 2. This case highlights how students can flexibly go back and forth between computing and mathematics to suit their immediate needs.

5.2.1 Tutorial 2: Logarithms and Taylor Expansions. The second tutorial asked students to write their own function for calculating logarithms using a Taylor expansion. The first part of Tutorial 2 gave students functioning code to calculate the Taylor expansion of the natural logarithm. Then, it asked them to implement a function to calculate the remainder for their choice of $a$, the point chosen as the basis for the Taylor expansion, and $n$, its number of terms. After having implemented a function that calculated the remainder, the students were given the opportunity to experiment with the two key parameters $a$ and $n$ and observe the results.

The tutorial's second part asked the students to exploit the binary representation of floating-point numbers in the computer to relax the accuracy requirements on using Taylor expansions to calculate logarithms. This is of particular interest because it mirrors how algorithms that calculate logarithms are made, even though approximations that require fewer terms than Taylor expansions are often used in practice.

The basic idea is as follows: Taylor expansions are usually intractable to use directly because they require a very large number of terms to reach the desired accuracy far from the fixed point $a$ one chooses as a basis to construct the expansion. However, by exploiting the way floating-point numbers are represented in the computer's binary memory, one may map the half-open interval of all positive real numbers to the closed interval of numbers between 0.5 and 1 .

This transformation is more impactful than it might appear at first glance. Achieving the desired accuracy for this small interval is vastly simpler than doing so for all positive numbers. This means that we can indeed use the computer's proficiency for working with basic calculations (such as polynomials) to represent more complex functions such as the logarithm.

At the end of the tutorial, the students were asked to implement their own log function in this way. With this done, the final task was for the students to make use of their earlier work on the remainder to pick optimal parameters for the Taylor expansion. The goal was that their log function would become machineaccurate on the one hand and as fast and efficient as possible on the other.

5.2.2 Overview of Case B. In Case B, we saw Rita and Lena use mathematics and computing flexibly. They attempted to use mathematics to find the optimal parameters for their program, and with support, they were able to find a numerical solution when an analytical one was not feasible. They went through Output 
Modelling and Math Implementation in that order, and the program closed the circle by transforming Code to Output. This demonstrates the existence of the circular process seen in Figure 13, which we have named Improvement Cycle:

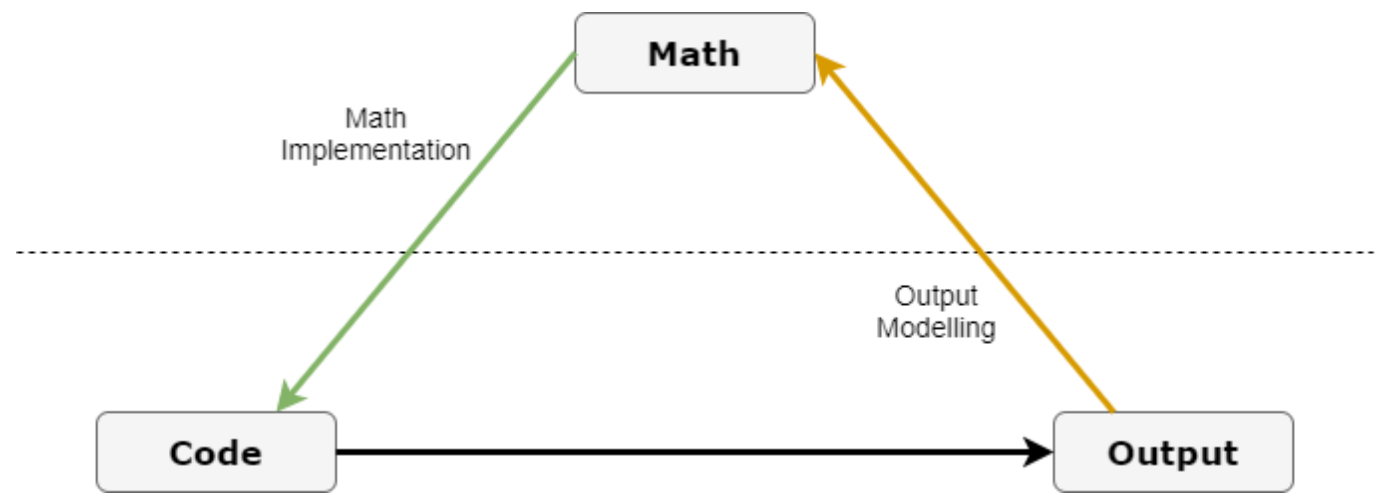

Figure 13: Improvement Cycle: Code produces Output, the students then use Output Modelling to get to Math, and finally Math Implementation is used to modify the Code further, as seen in Case B.

After completing an iteration of that cycle and being surprised by the result, Rita and Lena went back and forth between Math and Output to improve their previous solution and in the process were able to describe why their first choice was not optimal. This finally led them to choose parameter values that resulted in a log function that performed admirably in the test cases with many fewer terms than their initial attempt. Figure 14 illustrates this patten, which we have named Justified Improvement. This pattern differs from Improvement Cycle in that it only requires changes to the program input, not the Code itself.

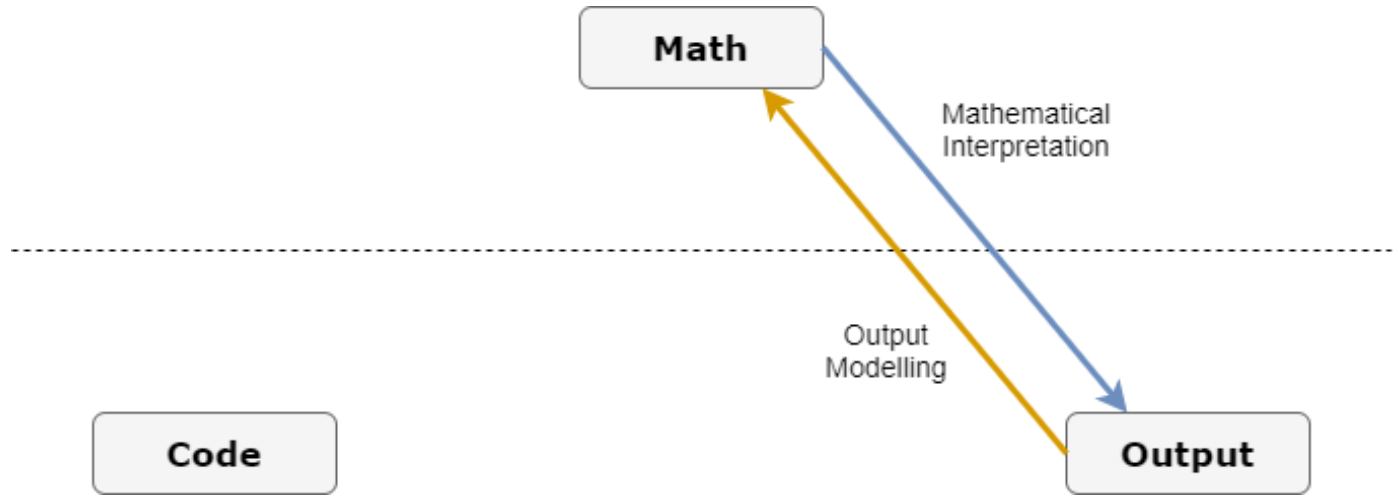

Figure 14: Justified Improvement, as seen in Case B.

In this interview, the students adhered to social distancing guidelines because of COVID-19 safeguards: Rita wrote on the whiteboard and Lena did all the typing on the computer keyboard. Lena had previously taken IT classes in high school, but these classes predate the recent initiative to introduce programming into the Norwegian high school curriculum. While some programming concepts are covered, these courses were very general, covering everything from databases to making web pages. Lena herself expressed that she did not feel those classes had prepared her very well for tasks such as the one in this tutorial. Rita had no prior programming experience, but she displayed familiarity and skill with many mathematical methods 
throughout the interview. Rita and Lena had already been interviewed together for an updated version of Tutorial 1 - this was their second interview together.

In this case, we use timestamps in the interview excerpts to indicate where they appear out of chronological order. This happens where we needed additional data from the follow-up questions at the end of the interview to make our point.

5.2.3 Improvement Cycle: Output Modelling. We begin our account of Rita and Lena in the final part of the tutorial. They had correctly calculated the remainder and completed writing their own logarithm function as described in the previous subsection. Their next task was to use the remainder to check that their choices of $a$ and $n$ had achieved the desired accuracy in the interval $0.5 \leq x<1$. As it turned out, their initial choices led to a much higher accuracy than the tutorial required (see Figure 15), which prompted the interviewer to challenge the students to find the minimum number of terms required for satisfactory accuracy. Rita and Lena briefly discussed whether they should engage in some form of trial and error or a more rigorous mathematical calculation.

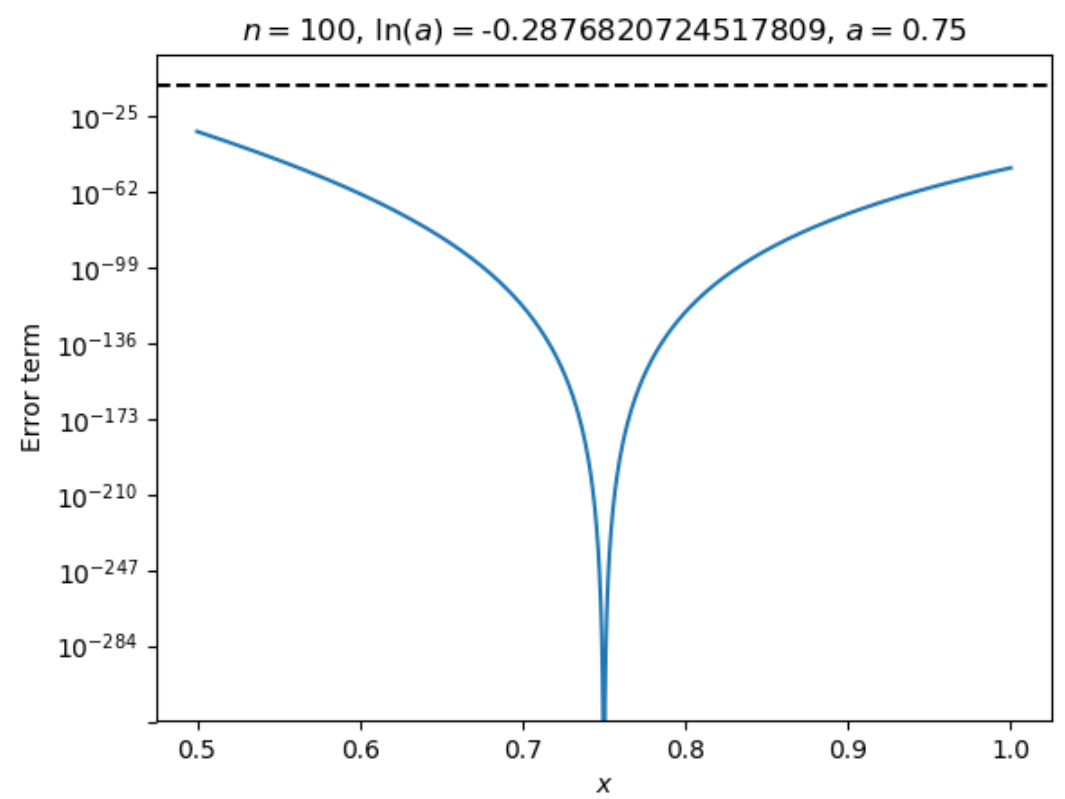

Figure 15: The students' initial results for $a=0.75$ and $n=100$ led to a much lower absolute value for the remainder than the stated goal of at most $10^{-10}$ in the entire interval (this threshold is represented by the dashed line in the plot).

Rita proceeded to ask Lena to open the document where the formula for the remainder was displayed. She then asked the interviewer whether they should try to solve that expression for $n$ or use trial and error instead. The interviewer conceded that the latter was the original intent, because there would have been a lot of $x$ values to check for otherwise.

Rita Oh yeah, no, but I thought that we just tested for 0.5 and 1 because that's the worst case.

Interviewer You wanted to test the endpoints where, sort of, it's the worst case?

Rita Yeah.

Interviewer That sounds like a really good idea to me. 
We interpret this exchange as Rita making a connection between the output (Figure 15, where the worstcase remainders at the endpoints are clearly visible) and solving an equation on the whiteboard. Rita did express some uncertainty at first but proceeded to write down an equation where the remainder was equal to $10^{-10}$, the limit for an acceptable remainder according to the tutorial. With Lena's help, she substituted the required values for $x=1$ into the remainder formula, resulting in an equation with $n$ as the only unknown (see Figure 16).

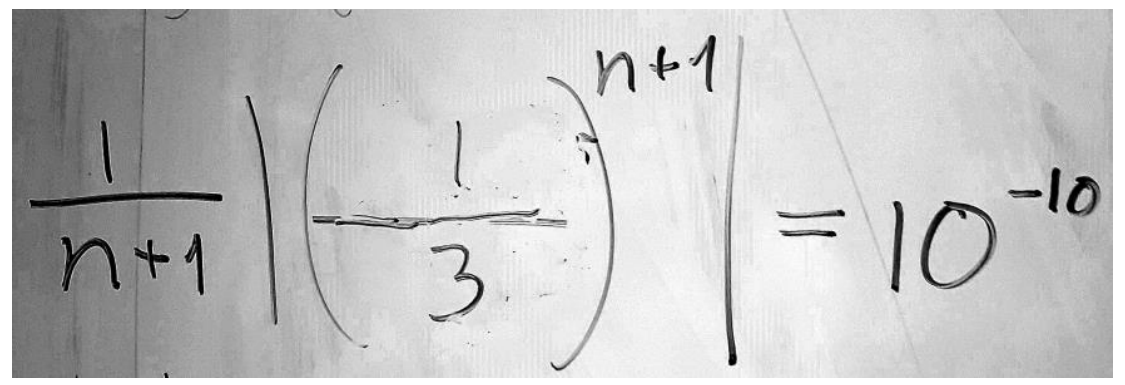

Figure 16: Rita's equation for the remainder at $x=1$ after substituting in the known quantities. The righthand side is the upper limit of an acceptable remainder, according to the tutorial.

This connection fits the Output Modelling label. Rita going to the whiteboard and stating that she wanted to perform the calculation analytically show us that the Target Domain is Math. We infer that the Source Domain is Output, referring back to the plot in Figure 15, where each side of the equation represents a line in that plot. We also note that Rita consulted a mathematical resource (the formula sheet that accompanied the tutorial) instead of looking at the code they had implemented earlier.

We can find supporting evidence in the follow-up questions at the end of the interview, when Rita and Lena talk about why they did the whiteboard calculations:

Lena [1:48:33] I think it was to get an overview, sort of. That it's easier to get it written down in a little larger...

Rita Yeah, I'm stronger... or I'm better at math than I am at programming.

Lena Yeah.

Rita So I often feel that if it's something that's calculable, I feel it becomes more correct when I do it mathematically.

Interviewer Or clearer, perhaps?

Rita Yeah, I kind of like having an expression for things.

From the student point of view, it appears that this Output Modelling afforded clarity and confidence in the result. It also afforded the use of familiar (to the students) mathematical tools like the substitution and simplification Rita made use of. This mirrors Gina and Benjamin's Code Modelling in Case A.

The students then spent some time manipulating this expression analytically on the whiteboard (see Figure 17), but eventually concluded that it would be difficult for them to solve. It turns out that their hunch

${ }^{5}$ She means analytically. The distinction was obfuscated in translation. 
was correct, as the expert solution involves a function called the product log function that is only implicitly defined as the solution to equations on this form (Wolfram Alpha, n.d.). Importantly, then, they reached a point here where they did not, according to Rita, "get an expression for $n$ ".

5.2.4 Improvement Cycle: Math Implementation. At this point, Lena found another way forward: "I was about to ask whether it'd be easier to write that formula here in Python or something. Whether we're able to calculate it in that case."

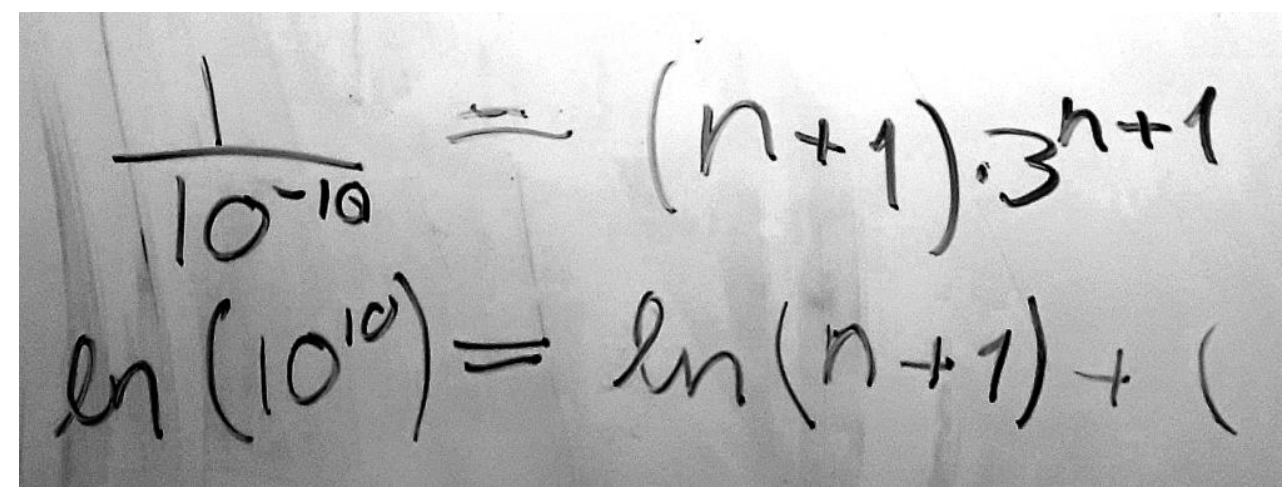

Figure 17: The endpoint of Rita's first whiteboard calculation.

At this point, Rita and Lena stated that the operations they saw as available to them would only make them come full circle, and to progress they would have to take another approach. We note with interest that Lena suggested Python without prompting on the interviewer's part. In the follow-up question part of the interview, she elaborated on this transition back to computing:

Interviewer [1:47:08] But how did you come up with "hey, we can do this in Python", Lena?

Because that was also sort of a breakthrough idea that helped us further.

Lena Yeah. No, really, the thing is that when there are very complicated expressions, or like when we have something raised to the power of 1000 or something like that.

Interviewer $\mathrm{Mm}$ - $\mathrm{hmm}$ [affirmative].

Lena Then you do quickly think that it can't be done by hand. But that it's quite easy to type. And then you get an answer. So then you save some time.

Both students stated for the record that they believed they would not have been able to find the optimal value of $n$ without programming.

After suggesting using Python, Lena proceeded to open the code editor and translated the equation on the whiteboard to the form of Figure 18, which contained no fractions or logarithms. It might be that Lena considered such a form to be the simplest one to solve, because this was not the form that they had written on the whiteboard in Figure 17. If so, Lena was in alignment with the expert perspective, except for getting Python's assignment operator $=$ confused with $==$, the evaluation of equality (see also footnote 6). Nonetheless, given Lena's suggestion to "write that formula here in Python" and the mathematical equivalence of the two expressions, we infer that she saw a similarity between them. 


\section{$1(n+1) * 3 * *(n+1)=10 * * 10$ \\ Figure 18: Lena's translation of the equation in Figure 17 to the code editor (for $x=1$ ).}

The students were unsure how to progress from there, however. They attempted to look for a Python function named solve() that would solve the equation for them analytically (see Figure 19), but the interviewer discouraged this approach due to time constraints and the requirement of using Python libraries unfamiliar to the students like sympy.

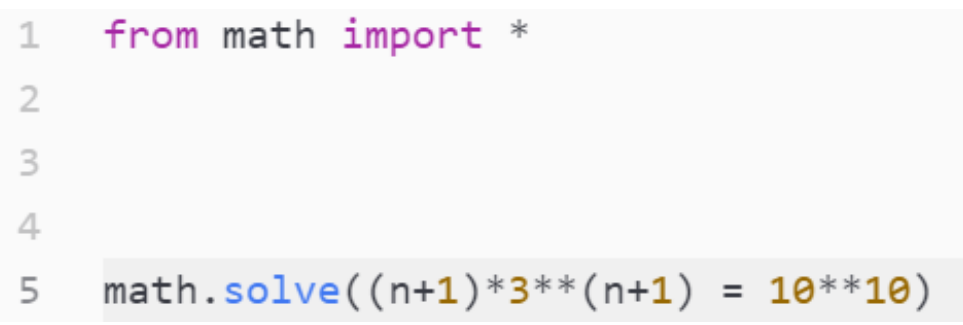

Figure 19: Lena's first attempt at a Python solution.

We classify this connection as Math Implementation. The Source Domain is Math, through Rita's earlier whiteboard work. The Target Medium and Syntax are Code, as the focus was on Lena's work on the computer at this point. As for the Target Structure, we claim that this represents the first stage in a two-step connection, much like Gina and Benjamin in Case A, with two important differences: (a) the Target Syntax is not a hybrid state containing elements of both Math and Code ${ }^{6}$, and (b), Lena's work is a computational representation of a mathematical entity, which is the opposite of what Gina and Benjamin made. Like in Case A, this would soon change as the interviewer intervened.

First, however, we note that the way the students first attempted to implement the problem was still very closely linked to their earlier analytical whiteboard work. They initially wanted the computer to do the exact same thing they had attempted themselves by hand. When that did not work, they did not seem to see how to connect the equation to familiar Python concepts on their own, perhaps due to their still interpreting it as something mathematical. Wanting to remain in the Python environment, the interviewer offered an alternative way to accomplish the task using Python:

Interviewer We could just try different $n$ values and see whether it becomes $10^{10}$, though.

Lena Yeah.

Interviewer In this one. But it could also be that there is a way to have Python try... a lot of different $n$ values for us. So that we don't have to try each and every...

\footnotetext{
${ }^{6}$ In theory, one could object that the use of single-letter variable names points us toward Math, but in Code these are perfectly legal. Furthermore, while shortening variable names so that they are not interpreted as the product of many variables $(c a t \neq c \cdot a \cdot t)$ makes sense when representing Code using Math, as Gina and Benjamin did, lengthening variable names makes little sense from an expert perspective, as there is no such ambiguity in Lena's resulting expression (Figure 18).
} 
Rita Oh, yes.

Lena For loop?

Rita If we write, like, while or for, yeah.

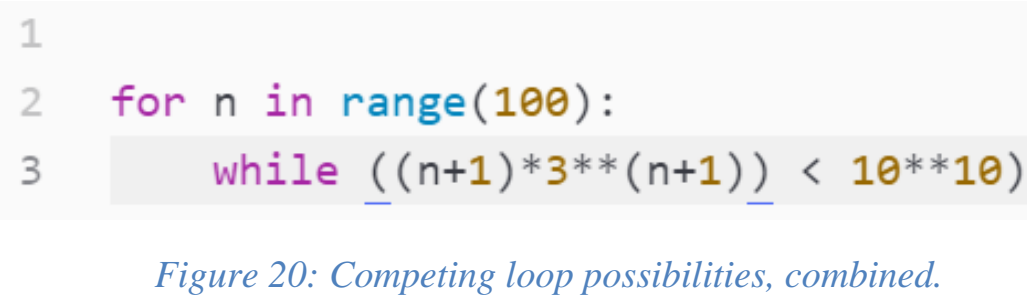

The interviewer's first idea ("try many different values") was enthusiastically received by the students, who immediately related this intentionally vague statement to the computational concept of loops. The interviewer's further suggestion that they use a while loop that would stop when the answer is good enough was immediately connected to and expressed as an inequality by Rita, which Lena then leapt at the chance to implement. In other words, they articulated a connection between the mathematical educated guess (picking a possible solution that seems reasonable and checking if that satisfies the inequality) and the computational iteration (trying out every possibility in ascending order until the inequality is satisfied).

This connection completes the transition into Math Implementation. The change from earlier is that the Target Structure seems to have changed from Math to Code at this point. This is based on the students implementing an inequality as the test condition that yields True or False, which is structurally different from simply enclosing an equation within math.solve() as they did earlier (in Figure 19).

The affordance of this connection is a better understanding of the process of determining the optimal value of $n$, which now features in a computational role as the loop variable in addition to being the mathematical unknown that is solved for. The additional affordance to leverage their coding experience without resorting to symbolic algebra libraries was not lost on the students. They explicitly rejected the idea of using the sympy library for the task at hand, given their lack of familiarity with it.

Instead, they started setting up two possible loop structures (while and for) and initially tried to combine them (see Figure 20), but eventually decided that the for loop was unnecessary and with some input from the interviewer on correct syntax arrived at a functioning while loop (Figure 21).

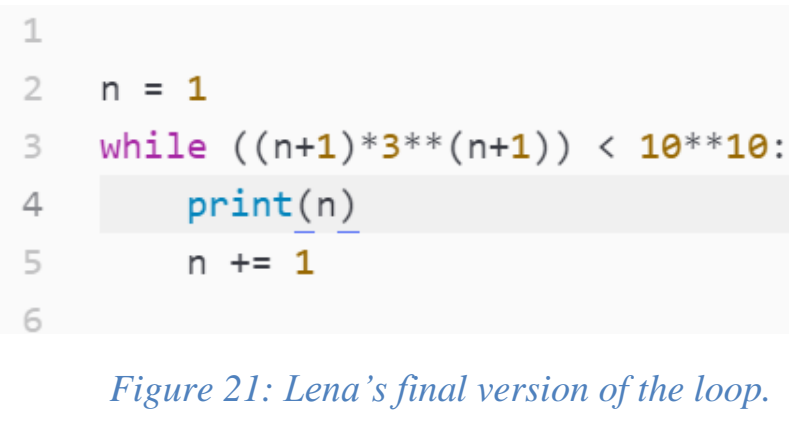

5.2.5 Improvement Cycle: Running the Code. At this point, the students tested their program, resulting in the output seen in Figure 22. The inequality was satisfied as long as the remainder was too large 
- in this case, the loop would continue. It stopped at the point where the remainder was acceptably small, hence the final printed value represented the minimum number of terms that produced an acceptable result:

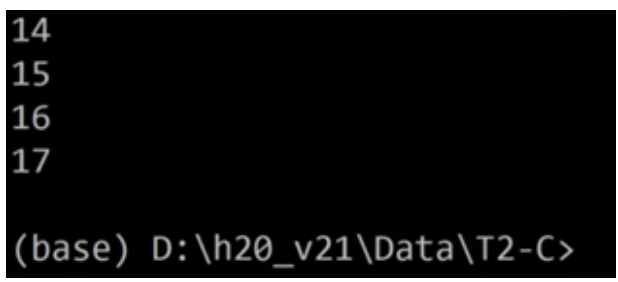

Figure 22: Output of the students' program for $x=1$, with $n=17$ as the number of terms that keeps the remainder sufficiently small.

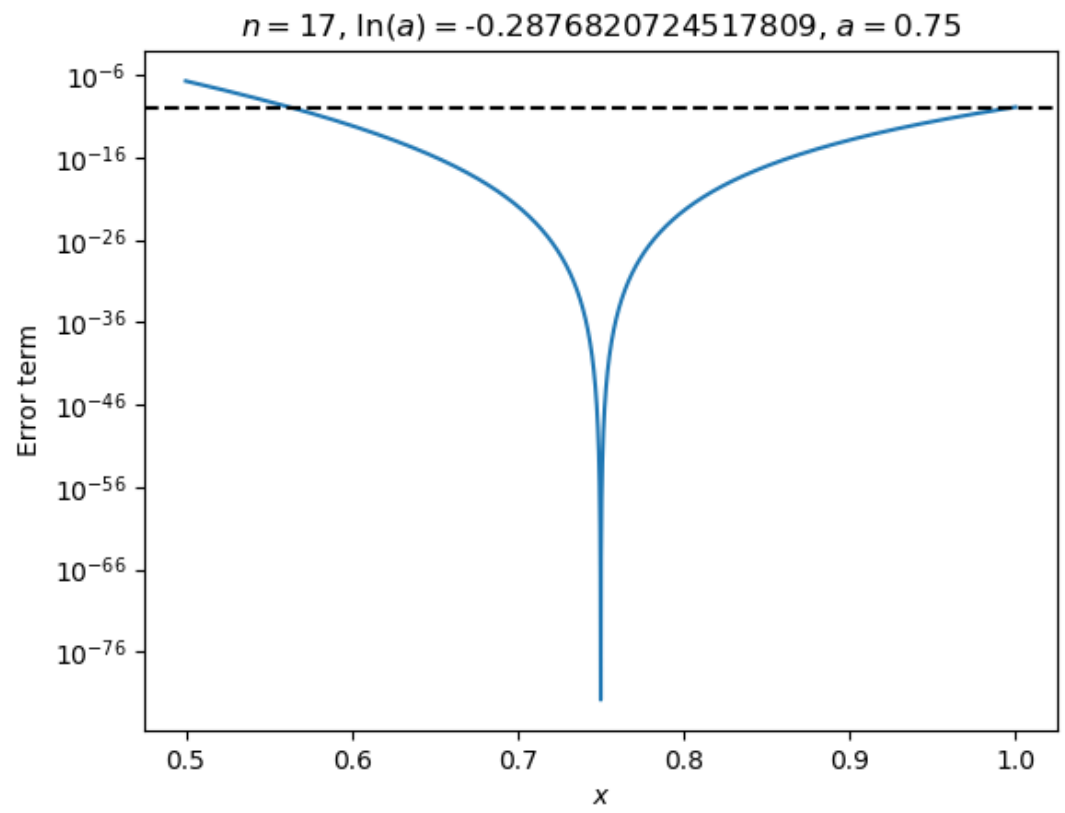

Figure 23: Plot of the remainder with $n=17$.

5.2.6 Justified Improvement: Mathematical Interpretation. Lena proceeded to modify the code the students used earlier, reducing the number of terms from 100 to 17, while keeping the fixed point $a$ of the Taylor expansion unchanged. Unfortunately, attempting to use the newfound minimal value $n=17 \mathrm{did}$ not result in the desired accuracy in the entire interval between 0.5 and 1 , as it proved to be insufficient for low values near $x=0.5$ (see Figure 23). The students' first reaction to the result suggested skepticism, as they remained completely stationary upon seeing the plot, and a significant pause followed before they spoke in a hesitant tone of voice. The students did not immediately buy into the interviewer's interpretation of the result as a partial success:

Rita Ehhh, yes. Right.

Interviewer I thought that worked well, actually.

Rita But, it's not below $10^{-10}$ though. 
Lena [inaudible] Yeah. How do you think that works well?

Interviewer Because I'm looking at the point 1. $x=1$. And there's it's sort of a hair's breadth below.

Lena Yeah.

Rita Oh yeah, that's what we calculated, yes. Yeah, because then we should do that for both sides, then. But that ... yeah. [The others voice agreement.] Because that did work well. And then you just take the largest $n$ value of the two endpoints.

We claim that while Rita was looking at the output (plot), she made another cross-domain connection from her earlier work on the whiteboard. She saw a similarity between the two endpoints of the curve and the two $x$ values she could have chosen for her mathematical investigation.

We label this connection Mathematical Interpretation, as the Target Domain was the Output that the students attended to, while the Source Domain was Math (Rita's choice of $x=1$ when setting up the original equation). This connection afforded validation of the result and making a better choice for the next attempt: one needs to look at the entire interval and choose the point that maximizes the remainder to guarantee that the remainder is not too large at any point.

5.2.7 Justified Improvement: Output Modelling. We conjecture that a fourth connection was made shortly thereafter when Rita proceeded to repeat the calculation for $x=0.5$ and the interviewer pointed out that the parameter $\xi=\min (a, x)$ in the remainder formula would also change because of the change in $x$. In other words, she saw a similarity between the value of this parameter in the formula and the magnitude of the remainder in the output.

This connection is another example of Output Modelling. We claim that the Source Domain Rita is referring to would be Output (the plot in Figure 23), where the fact that $x=0.5$ had a larger remainder was evident. The Target Domain is Math, as Rita was working with the new equation at the whiteboard at this point. This connection then afforded explaining why changing the value of a variable had a particular effect on the output: "because then you're dividing by a smaller number, and then it does become larger."

She then completed the calculation with these new values on the whiteboard and arrived at the result in Figure 24, which Lena then used as the basis for modifying their Python program. She then used the output (Figure 25) as an input parameter to the remainder function, resulting in the plot of Figure 26.

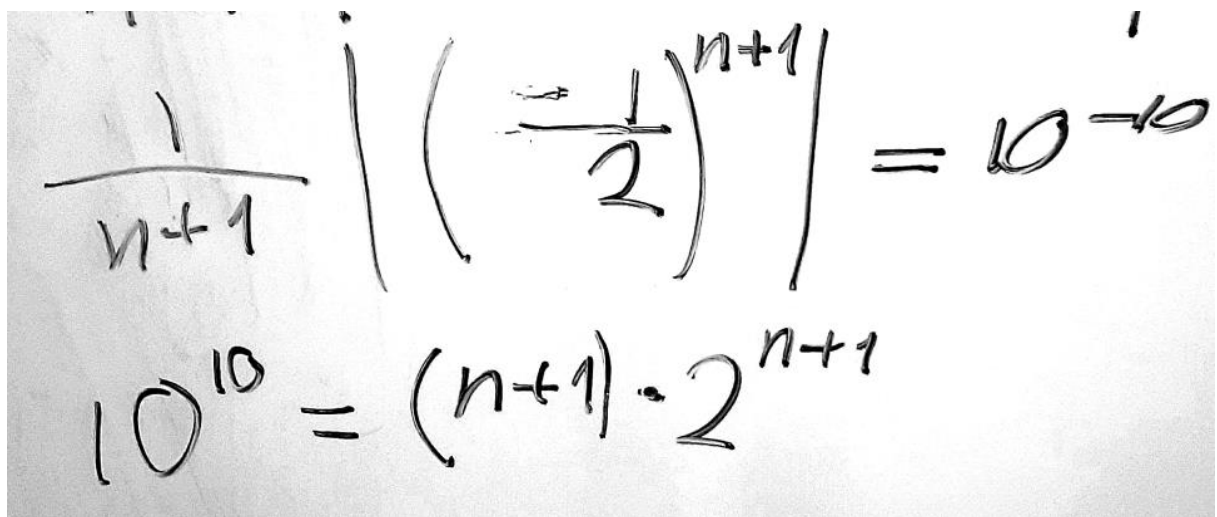

Figure 24: Rita's calculation for $x=0.5$. Note the similarities with the expressions in Figure 16 (top) and Figure 18 (bottom). 


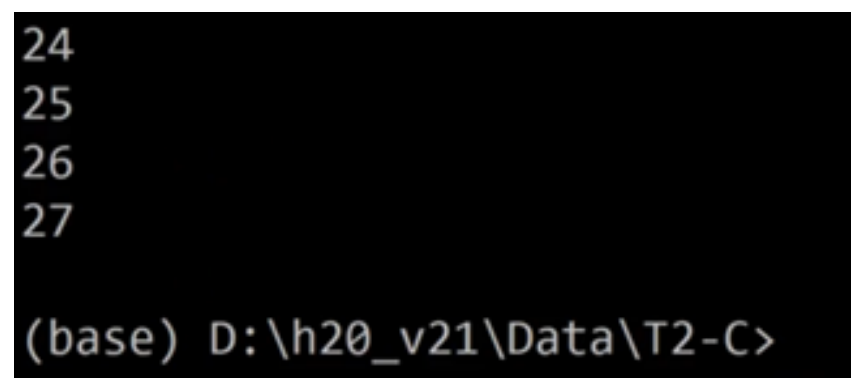

Figure 25: Output of the students' code for $x=0.5$.

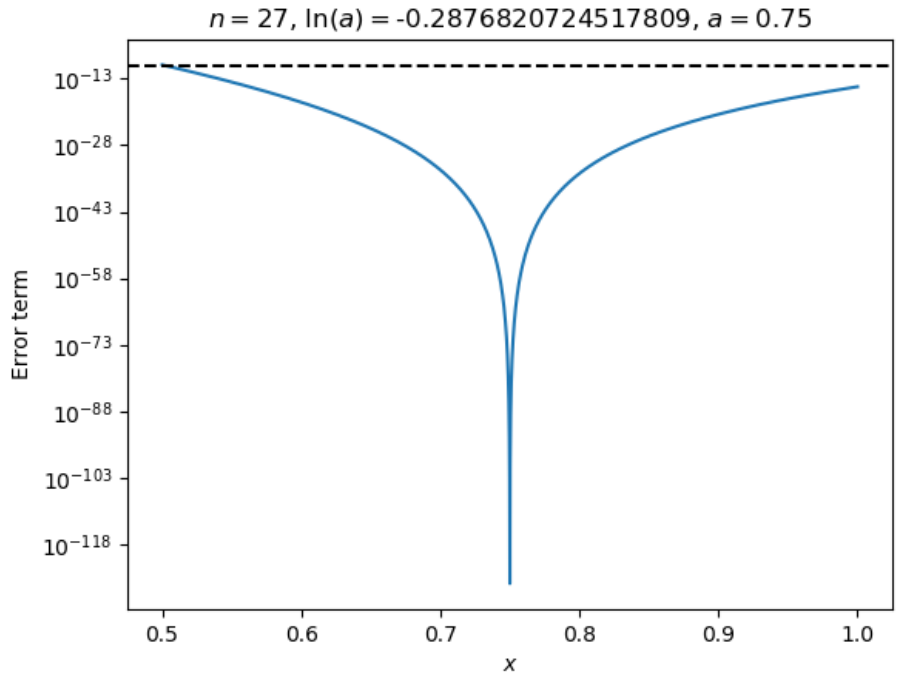

Figure 26: Result of using the number of terms in Figure 25.

Upon seeing the result in Figure 26, the interviewer and the students expressed satisfaction:

\section{Interviewer Wow.}

Rita Shit, we're smart.

Lena [laughs] Yeah.

As a final test, Rita and Lena used $a=0.75$ and $n=27$ as input to their logarithm function. A comparison between the commonly used numpy library's log function and the students' own results can be found in Figure 27. As the students pointed out, all the digits were identical, and the relative error was zero, as seen in Figure 27:

To sum things up, the students were faced with the dilemma of how many terms to include in the Taylor expansion. They connected that question to a mathematical equation. When this failed to provide an analytical answer, they further connected the equation to a numerical trial-and-error approach using a loop. At this point, the equation had been transformed into a form that made it simpler to use numerically, with no need for a function to calculate the absolute value, for instance. From an expert perspective, one could argue that the students could have done this without the mathematical work, but the fact that the students used the results of their work indicates that it had value to them. 
5.2.8 Summary of Case B. In short, the students went from Output to Math and then to Code. The computer then closed the circle by taking the Code and producing new Output. The students then made further connections by using mathematics to explain what had happened and decide what to do next when the plot surprised them. As they re-did the whiteboard calculations, they referred to the plot and explained what happened to the remainder using mathematics. This second set of connections thus took them from Math to Output and back to Math again.

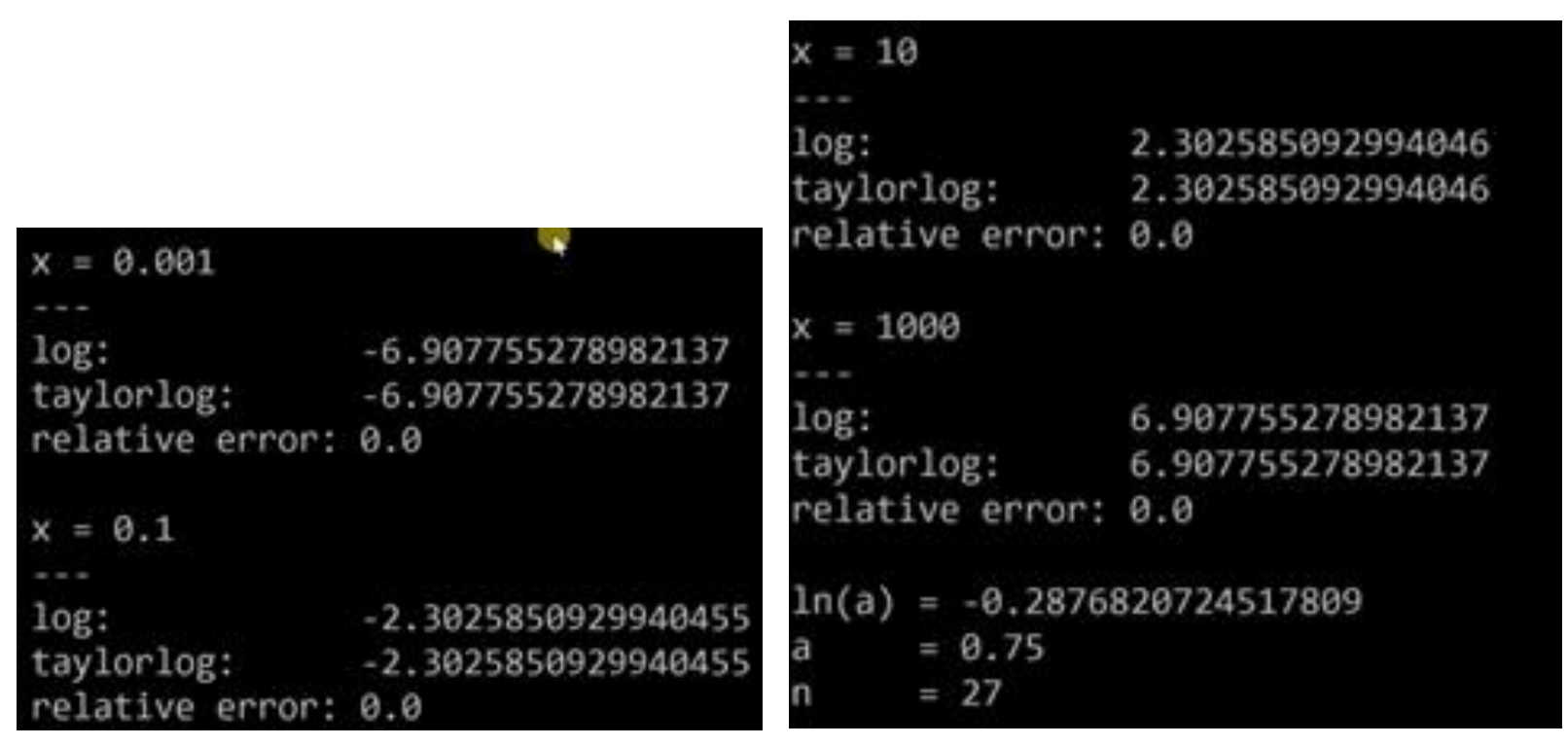

Figure 27: Tests of the students' own log function.

What these connections have in common is that each of them afforded a new perspective that provided deeper insights into the problem. The guessing game to find the ideal value for the parameter $n$ transformed into a rigorous mathematical solution with the potential for greater explanatory power. The realization that the equation was hard to solve analytically transformed into a systematic way to find a numerical solution. They were now able to explain why $x=0.5$ was the better choice, not just that it was better than $x=1$. And finally, they were able to justify their choices of parameters as ideal with regards to calculating the logarithm with as few terms as possible.

\subsection{Case C: Lydia, Martin, and Roger (Tutorial 3, 2019)}

In our final case, we present work by Lydia, Martin, and Roger on Tutorial 3. This case highlights how a task that asked the students to implement mathematics on the computer facilitated fruitful mathematical discussion.

5.3.1 Tutorial 3: Numerical Integration. The final tutorial had students numerically integrate a familiar function whose integral value is well known but impossible to calculate analytically: the standard normal distribution. The students were tasked with implementing a function that calculated one term in the Riemann sum using the midpoint method (they were given code that would then use their function to calculate the entire integral).

It is worth noting that the midpoint method with step length $h$ they were asked to use, 


$$
\int_{a}^{b} f(x) d x=\sum_{x=a}^{b} f\left(x+\frac{h}{2}\right) \cdot h
$$

can be interpreted as a special case of the more general Euler's midpoint method for differential equations (Mørken, 2017),

$$
\begin{aligned}
& x^{\prime}=f(t, x) \\
& x_{k+\frac{1}{2}}=x_{k}+\frac{h}{2} f\left(t_{k}, x_{k}\right) \\
& x_{k+1}=x_{k}+h \cdot f\left(t_{k}+\frac{h}{2}, x_{k+\frac{1}{2}}\right)
\end{aligned}
$$

Note that in the latter case the symbol $x$ has taken on a different meaning (a function) than in the former (where it is the independent variable). Expressing the integral midpoint method with differential equation notation would look like

$$
\int_{a}^{b} f(t, x) d t=x_{0}+\sum_{k=1}^{n} x_{k}=\sum_{t=a}^{b} f\left(t+\frac{h}{2}\right) \cdot h
$$

The reason for this is that in the special case of the integral, $f(t, x)$ has no dependence on $x$ and the boundary condition is $x_{0}=x(a)=0$. It is perhaps not surprising, then, that the students in Case $\mathrm{C}$ would confuse the two and treat these as two unrelated methods. They really do look different from one context to the next.

The central question in this tutorial was how many steps (terms in the Riemann sum) would be needed to balance accuracy with efficiency. After being encouraged to try out some values and note the results, the students were asked to plot the relative error as a function of the number of steps. The goal was that they should discover a sweet spot, where the mathematical error is as small as possible and the rounding errors (that increase with more terms) also having minimal effect.

5.3.2 Summary of Case C. In Case C, we saw Lydia, Martin, and Roger resort to mathematical drawing as a step in the process of implementing the code for numerical integration. They went back and forth between Code Modelling and Math Implementation. While they finally used the Output for verification, it did not feature in their subsequent mathematical discussions in this case, in contrast to Case B. We have named this pattern Justified Design and illustrate it in Figure 28. 


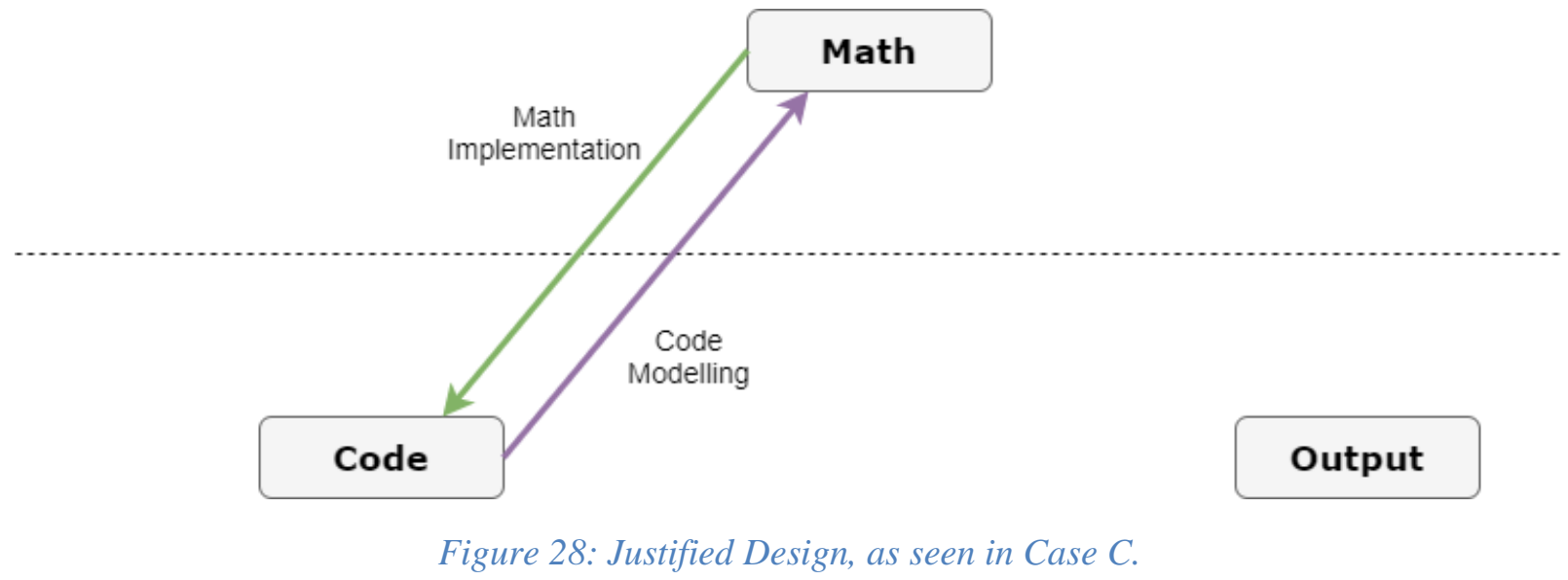

Lydia was verbally active and asked several questions throughout the interview. She often asked the group to pause their current activity to discuss the underlying concepts. Martin stated that he had previous experience using Python from work, and Lydia and Roger often deferred to him in times of discussion. Martin also typically initiated the writing or typing on the keyboard. Roger's interactions with the group mostly concerned completing the given tasks. The three students were used to working together from the group sessions in class.

5.3.3 Code Modelling. Lydia, Martin, and Roger had just been asked to implement the midpoint method for numerical integration in Python (not the complete integral, but a function that returned a single term in the Riemann sum). Lydia's first reaction was to mention that this sounded like Euler's method and suggested they "run it by hand first". This choice of words is interesting and suggests that Lydia saw a similarity between the task of writing the method and performing the same procedure on paper.

We interpret this connection as another example of Code Modelling. Martin ended up making a drawing of the function plot with boxes representing steps in the integration, as seen in Figure 29, hence the Target Domain is Math. The Source Domain is the Code, namely the function they were asked to design and implement. They had opened the code editor before Martin started drawing.

In contrast to Case A, the students were not deciphering a piece of code they had been given but were instead asked to write and implement the code themselves. They also used a mathematical drawing rather than setting up an equation to solve. Nonetheless, Lydia's comment about "running it by hand" reminds us of Gina's "by hand" comment in Case A. Unlike that comment, however, Lydia explicitly blended a computational construct ("running") with a mathematical one ("by hand"). The possibility that something written by hand could be run supports our interpretation that Lydia was aiming at Code Modelling much like it played out in Gina and Benjamin's case. 


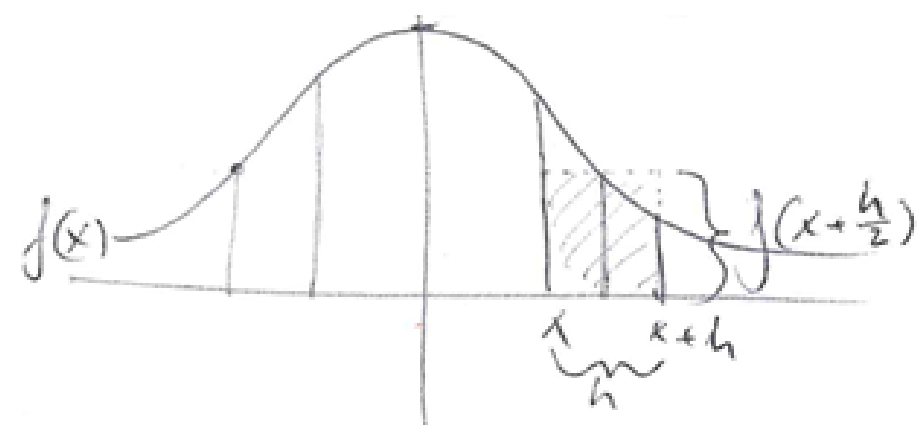

Figure 29: The drawing from Martin's worksheet. This version of the drawing is more complete than it is when it is first referenced in the transcript (Martin refined this drawing several times).

After completing the first version of the drawing, computational ideas kept emerging in the students' conversation:

Roger What was $n$ ?

Martin $n$ is the number of points we've been given...

Roger Yes, $O K$.

Martin ...or the size of the precision.

Lydia Now you're multiplying [inaudible] along...

Martin Ehm, does anyone remember the midpoint method?

Lydia Yes, first we must go a half-step [points to Martin's drawing].

Martin Yes [continues drawing].

Lydia Ehm... and then we have to store that variable.

In this conversation, we note that the students used language that referenced not only computational concerns, but also specifics of the implementation. Computational elements were re-introduced when Roger asked about a computational variable ( $n$ ) that was not present in the drawing. Moreover, Lydia's explanation of the midpoint method explicitly referenced storing a result in a variable, which would be an unconventional way of putting it in traditional mathematics. It is common that students relate situations that experts would not (see for instance Lockwood, 2011), but taken together with Roger's question, we find it more likely that the context of the program they want to write was on the students' minds as they did the mathematical work.

It is possible that this is what Lydia meant earlier by "running the code by hand": doing a calculation on paper while at the same time imagining what it would take to implement it on the computer and considering the process of that implementation. We find this to be consistent with our idea of Code Modelling: the students are engaged in mathematics while noticing similarities to the task of writing a program.

What this connection appeared to afford the students was, much like in Case A, a visualization of the code and method they were asked to implement. Unlike Gina and Benjamin, these students did not apply mathematical tools like substitution and equation solving. Instead, they used Martin's drawing as the basis for a mathematical discussion about the midpoint method and its relation to other concepts, and this discussion continued throughout the interview.

5.3.4 Math Implementation. The discussion of how to implement the midpoint method initially lasted until Martin recognized that they were ready to return to the code editor: "We are able to calculate that." 
At this point, Martin began modifying the Python code, as shown in Figure 30. We interpret this as Martin seeing a similarity between his drawing and the code he was writing.

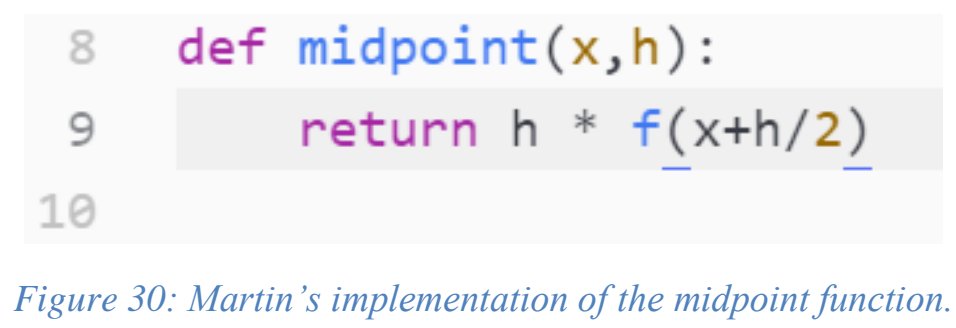

Martin thus produced a computational function to represent a mathematical function ${ }^{7}$ and chose variable names so that the computational variables represented their mathematical counterparts. This could suggest that the Target Syntax is Math, but the variable names $x$ and $h$ were given in the tutorial itself and lengthening them would be uncommon (see footnote 10 in Case B). Furthermore, failure to make the Target Syntax Code here would likely have led to error messages.

The Target Domain is Code, because Martin was writing the function in the code editor ${ }^{8}$. His utterances as he wrote the function strongly suggested that the Source Domain is Math. His claim that "we are able to calculate that" also connects the code he subsequently wrote to the earlier mathematical midpoint method discussion between the students, at which point he was focused on his drawing.

This suffices for us to interpret this connection as Math Implementation, which suggests to us that the variables were intended to be Code representations of the original Math variables. What this connection afforded was an implementation of the method they had been recalling and describing. We note that while doing Code Modelling the students were attending to the particulars of the implementation, but they ended up using none of the particulars they discussed (the number of terms and storing the result of a half-step in a variable). Even so, we claim that this Code Modelling laid the foundation for the next connection that followed.

The students then evaluated the work to see if what Martin had done made sense. Martin asked the interviewer (who had informed the students that he could be used as a TA in this setting) a question:

Martin [to interviewer] Is this the same as the Euler midpoint method, where he takes a half step to find one point and its derivative and then he takes the derivative...

Lydia [draws parallel, slanted lines in the air with her hand] and then he makes another one below that is parallel with the other line [laughs] where everyone reacted to [the instructor] drawing it very badly... heh.

We interpret this exchange as Martin and Lydia seeing a similarity between the code in Figure 30 and a mathematics lecture that they remembered attending. We classify this as Math Implementation, since the lecture took place before they wrote the code. To see what the other students made of the question, the interviewer withheld his answer until the discussion came to a stop, which did not occur. Instead, the

\footnotetext{
${ }^{7}$ This distinction is perhaps not obvious. While a mathematical function is a one-to-one mapping between two sets of elements (typically numbers), computational functions can perform tasks that have little to do with the mapping between input and output, such as writing to the screen or saving to a file. It is therefore prudent to regard mathematical functions that can be represented computationally as a subset of all computational functions.

${ }^{8}$ It would be uncommon, but possible, to use the code editor as a sketchpad to solve an equation analytically. That would make the Target Structure Math, as opposed to what we describe here.
} 
students confirmed that their implementation gave the result that they were supposed to get (as indicated in the tutorial) by running the program.

At this point Martin and Lydia both seemed to be seeking to reconcile the method they just wrote with what they learned in the recent lectures on Euler's method. Roger seemed less concerned as long as what they did both worked and made sense in itself. Martin then expressed his belief in there being two different midpoint methods, and Lydia noted that the one they had just implemented seemed much easier to use than the method she remembered from the lectures. Finally, Martin made a connection that allowed them to distinguish between the two methods: "No, [Euler's midpoint method is] for differential equations, of course!"

This represents something of a breakthrough for the three of them and brought relieved laughter as it solved the mystery the students had been grappling with. We claim that the realization that there were two different midpoint methods (from their point of view) and the subsequent categorization of their uses both sprung from the computational task of implementing the method for numerical integration. The same can be said for Lydia's successful attempt to categorize the method they had used, which she explicitly stated that she recognized as Riemann sums.

5.3.5 Summary of Case C. To summarize his episode, this group of students went back and forth between Math and Code as they designed their function and only subsequently made use of the Output to determine its correctness. Finally, they took a step back from the code to a mathematical discussion which helped them see similarities between their work and earlier lectures.

\subsection{Synthesis of Connection Patterns}

In these three cases, we have seen students make connections between mathematical and computational ideas as they worked with tasks that integrated knowledge from these two domains. Each case demonstrates a distinct way that students connect these ideas, often in ways that they had not done earlier in the interviews, or in ways that were not anticipated by the tutorial designs. Our cases also represent mathematical contexts in which mathematics and computing were integrated, and these exemplify the kinds of situations in which such integration may be leveraged and explored.

This subsection provides summaries of the four unique patterns that the cases exemplified. These patterns and their affordances are summarized in Table 3. The affordances we link to each pattern in that table are taken from our analysis of the connections as they appeared in the analysis in Section 5.

Table 3: Summary of Connection Patterns.

\begin{tabular}{|c|l|l|l|}
\hline $\begin{array}{c}\text { Pattern } \\
\text { Program }\end{array}$ & Definition & Case & Main affordances \\
\hline $\begin{array}{c}\text { Code Modelling } \rightarrow \\
\text { Mathematical Interpretation } \\
\text { Cycle }\end{array}$ & $\begin{array}{l}\text { A } \\
\text { Math Implementation } \\
(\rightarrow \text { running the program) }\end{array}$ & $\begin{array}{l}\text { Understanding a program } \\
\text { Formal proof }\end{array}$ \\
\hline $\begin{array}{c}\text { Justified } \\
\text { Improvement }\end{array}$ & $\begin{array}{l}\text { Mathematical Interpretation } \\
\text { and } \\
\text { Output Modelling (in any } \\
\text { order) }\end{array}$ & B & $\begin{array}{l}\text { Output closer to standards } \\
\text { Explaining process }\end{array}$ \\
\hline
\end{tabular}




\begin{tabular}{|l|l|l|l|}
\hline Justified Design & $\begin{array}{l}\text { Math Implementation and } \\
\text { Code Modelling (in any } \\
\text { order) }\end{array}$ & C & $\begin{array}{l}\text { Confidence in correctness } \\
\text { Organizing mathematical knowledge }\end{array}$ \\
\hline
\end{tabular}

Note that all the patterns, except for Replicating Program, were inherently cyclic in our data. Because they ended up where they began, students might be able to repeat these patterns for several iterations, if necessary, without additional changes of activity. It is possible that an extra connection from Output to Code could make Replicating Program cyclic in the same way. We do not have sufficient evidence to support this possibility at present, however.

As we saw in Case A, through manipulating and reasoning about mathematical equations, the students were able to construct a formal proof of the program's intended function. If both had recognized this proof for what it was, we suspect the resulting comparison between the proof and the output of the program would have provided them with a rich opportunity to identify the problem with the program and to discuss numerical problems versus mathematical problems in general.

This implies that making these kinds of cross-domain connections can lead students to gain profound insights about Code through thinking of it as Math, especially when it comes to information that is implicitly present in the code and requires mathematical thinking to unpack. In any case, their Code Modelling supported the students' work, because familiar algebraic manipulation highlighted a feature of the problem that they (correctly) perceived as relevant.

In Case B, the students initially went through an Improvement Cycle where they did mathematical work on the basis of program output and then wrote a new program to solve the mathematical problem they had formulated. This afforded them to be able to explain how to solve their equation numerically. Subsequently, they performed Justified Improvement to improve their results, which in turn enabled them to justify why one endpoint was a better choice than the other. Both patterns contributed to producing program output that was closer to the standards laid out by the interviewer (as many Taylor terms as required, but no more).

In Case C, the Justified Design pattern enabled the students to discuss the mathematical method the program was supposed to represent and revealed a conflict between their work and what they remembered from the lectures about Euler's midpoint method. The resolution came with the realization that there are two midpoint methods that they had confused, and the case ended with the students reinforcing connections between what they were doing and their mathematics classes. The pattern thus afforded them to think more deeply about the math they had learned and their organization of that knowledge.

What these cases show us is that working with mathematics and computing in an integrated way has the potential to support students' mathematical reasoning and organization of knowledge in powerful and flexible ways. These affordances can be seen as a direct result of making cross-domain connections. For a certain class of problems, we have demonstrated that it is possible to design for connections between these domains.

\section{Discussion and Conclusions.}

In this paper, we have categorized students' cross-domain connections according to the classification scheme in Table 2, all of which are based on connections we saw in our data. These connections demonstrate how students see similarities across domains and find these relevant enough to merit a shift in activity or use resources from different domains simultaneously. Our students used mathematics as a resource to write and understand code, and to interpret output. Conversely, they used both code and output as the basis for doing mathematical work that helped them understand and modify their computer programs. These connections match the descriptions given by Høffding (1892) and Lobato and Siebert (2002). 
Additionally, we recognize Karakok's (2019) flexible shifts between multiple modes of thinking in these connections: Code, Output and Math are the labels we have ascribed to these modes. In Case A we see Gina and Benjamin treat the whiteboard work differently when interpreting it as a representation of the code as opposed to when they treat it at something inherently mathematical. While their shift was perhaps not as flexible as we could have wished for, requiring the interviewer to re-frame the activity for the full connection to be made, the elegant proof they produced suggests that supporting students in becoming more flexible between these modes is something that is worth investigating further. There might well be more modes (subdomains) than we have encountered here, or modes of an even finer grain size, that are useful to us.

What is computing good for in a mathematical context? A clue might be taken from Greeno et al. (1993), who discussed affordances for student reasoning in particular. Even if students arrive at similar answers without using computers, our results suggest that student reasoning is affected by these shifts between modes of thinking, and not adversely. Our students were able to reason by proving that a program works, explaining both the process of the program's logic and particular results that it produced. This seems to support the suspicions of (2001) that algorithmic reasoning in mathematics and science differ from algebraic reasoning in fundamental ways.

Additionally, we saw students organizing their mathematical knowledge as a result of reasoning mathematically to write a simple piece of code, suggesting that there is potential value in writing algorithms so that computers may understand them. Of course, it is possible that shifting between modes also adds cognitive load to the students, and for inexperienced programmers especially, this potential may not be immediately accessible to novice programmers in a mathematical context.

The affordances we saw in the interviews appear to be linked to connection patterns rather than individual connections themselves, suggesting that what a single connection affords may depend on the other connections it forms a pattern with. If our analysis is correct on this point, our connection patterns in Table 3 represent a grain size that is useful for discussing affordances. We still rely on isolated connections in order to identify these patterns, however.

One such pattern, the circular process we called the Improvement Cycle is particularly interesting. This pattern suggests that students might be able to iteratively cycle through a pattern several times as they work toward increased understanding and a better-working program. There is a striking resemblance to the cycle depicted in p. 15 of (Kaufmann \& Stenseth, 2020), where "Use mathematics" and "Make hypothesis" could be interpreted as Math, "Change program" and "Test" as Code, and "Observe and analyze" as Output. In Case A and Case C, then, we have seen possible extensions to Kaufmann and Stenseth's model.

We also note a similarity between the connection patterns in Table 3 and the development process first depicted in Figure 3 of (Buteau \& Muller, 2010). We recognize that our Improvement Cycle and Justified Design appear in that figure as "Refining Cycle" and "Designing Cycle", respectively. The lack of connections from Math to Output means that Buteau and Muller's model does not contain Replicating Program and Justified Improvement, however. Therefore, in addition to more closely examining the individual connections and the affordances of patterns, our patterns can be interpreted as an extension to this model as well. (The "Programming Cycle" linking Code to Output in Buteau and Muller's model is not cross-domain and therefore not considered in this paper.)

In Section 2.1, we noted that domains like computing and mathematics are not disjoint but overlap at several points. One would expect that when computational and mathematical representations are similar, making connections between them would be easier from a student point of view. Case in point: when Gina and Benjamin wrote pseudo-mathematical statements to represent the code we gave them, these statements formed the basis for their re-interpretation of the code as mathematical, which in turn helped them mathematically prove that the program should work as advertised in the absence of rounding errors. 
Finally, we can point to some implications of these findings for instruction. We have demonstrated that it is possible, but not effortless, for students to reason by integrating knowledge from computing and mathematics. Teaching design enters into this, but it can also, as we saw, be helpful for the instructor to help students reframe a computational activity as mathematical and vice versa. It is possible that the connection patterns may even form a basis for learning activity design, aimed at the affordances found in this paper.

\subsection{Limitations and future directions}

One technical issue for the authors was that only the first and last author were native Norwegian speakers, and since the interviews were conducted in Norwegian, that meant half of the authors needed to rely on the first author's translations. This issue was circumvented as far as possible by careful review as indicated in Section 3.2, where any possible ambiguities were discussed and resolved among the authors. We also invited the students that we quoted to comment on the translated quotes. Nonetheless, we acknowledge the necessity of translating the raw data as a limitation.

It is possible that the patterns we observed were primarily due to the tutorial designs and interviewer intervention. We acknowledge this possibility, and it remains to be seen how much of what we observed was due to the task, prompting by the interviewer, and spontaneous contributions by the students, respectively. While we claim that the patterns in Section 5.4 exist and provide affordances for students in some circumstances, we do not claim that they occurred spontaneously. More likely, we have taken a first step toward describing contexts in which the patterns surface, which is useful if we find it desirable that students work in these ways.

We acknowledge that our data set is restricted to a relatively small number of students and contexts, and thus there are some limits to what we can claim. Further research is needed to uncover other possible connection labels and patterns we did not see in our data, and the fact that our classification scheme in Section 4 relies on expert knowledge might have caused us to miss some of the students' more exotic connections. We therefore take care not to claim that we have produced an exhaustive list of all possible connection types and patterns. We also see a potential for unpacking the causal links between teaching design principles and students' thinking in this context, and we will examine this more closely in a future paper (Sand et al., 2021).

It would also be prudent to look more closely at limitations and hindrances to students realizing the potential represented by these tutorials. Our data suggests that students who have insufficient prerequisite knowledge may struggle with making these kinds of connections unless they are supported by other students or teachers who can help them apply this knowledge in learning situations. With the present study in mind, one could certainly go looking for instances in which students failed to connect computing to mathematics: even though these events were not the focus of this paper, such failures did occur in our interview data.

Our data from Case A suggest that some students, especially when they are new to programming, may believe that their programs cannot make use of implicit knowledge - all the knowledge available to the program must be there in plain sight. If that were true, it would prevent programs from making use of knowledge that is implicit, such as mathematical knowledge, which may limit the possibilities that these students see. What caused this belief and how to correct it is another direction of future research that may prove useful.

More generally, we see potential in investigating students' conceptions of what is possible in mathematics, programming, and the integration of the two. In Section 4, we noted that we relied on the expert perspective when categorizing what domain an activity belonged to. In future studies, we would be interested in probing how the students themselves experience something as belonging to the domains of computing and mathematics. 
We also note from our data several instances in which the separation between Math and Code is not so clear. For instance, we had Gina's whiteboard model from Case A, before the students made the full transition to thinking of it as Math, or Lena's initial attempt at solving the equation with Python from Case $\mathrm{B}$, before the students made the connection to loops. We suspect that these "halfway points" will be important stepping-stones for many students in making cross-domain connections and we see potential in investigating these further, both from an education research and a teaching point of view.

In conclusion, we posit that an approach to teaching that integrates mathematics and computing has the potential for making powerful mathematical ideas tangible for learners in ways that do not diminish their richness or relevance. With this study, while we have contributed several examples of such by first-year university students, we cannot claim that our findings are exhaustive. We expect many more such examples to emerge through research and teaching practice in the coming decade.

\section{ACKNOWLEDGEMENTS}

This study was funded by the Norwegian Agency for International Cooperation and Quality Enhancement in Higher Education (DIKU), which supports the Centre for Computing in Science Education. We thank Linn Rykkje for very helpful comments on the manuscript.

Benton, L., Hoyles, C., Kalas, I., \& Noss, R. (2017). Bridging Primary Programming and Mathematics: Some Findings of Design Research in England. Digital Experiences in Mathematics Education, 3(2), 115-138. https://doi.org/10.1007/s40751-017-0028-x

Benton, L., Saunders, P., Kalas, I., Hoyles, C., \& Noss, R. (2018). Designing for learning mathematics through programming: A case study of pupils engaging with place value. International Journal of Child-Computer Interaction, 16, 68-76. https://doi.org/10.1016/j.ijcci.2017.12.004

Broley, L., Caron, F., \& Saint-Aubin, Y. (2018). Levels of Programming in Mathematical Research and University Mathematics Education. International Journal of Research in Undergraduate Mathematics Education, 4(1), 38-55. https://doi.org/10.1007/s40753-017-0066-1

Brown, B. (2017). Mathematics-How do I teach “math equality” vs. "CS assignment" vs. "CS equality logic operator"? Computer Science Educators Stack Exchange.

https://cseducators.stackexchange.com/questions/1300/how-do-i-teach-math-equality-vs-csassignment-vs-cs-equality-logic-operat

Brown, J., Stillman, G., \& Herbert, S. (2004). Can the notion of affordances be of use in the design of a technology enriched mathematics curriculum? In I. Putt, R. Faragher and M. McLean (Ed.). Mathematics Education for the third millennium: Towards 2010, Proceedings of the 27th annual conference of the Mathematics Education Research Group of Australasia. (pp. 119-126).

Buteau, C., \& Muller, E. (2010). Student development process of designing and implementing exploratory and learning objects. In V. Durand-Guerrier, S. Soury-Lavergne, \& F. Arzarello (Eds.), Proceedings of the sixth congress of the European mathematical society for research in mathematics education (pp. 1111-1120). http://ife.ens-lyon.fr/publications/editionelectronique/cerme6/wg7-07-buteaumuller.pdf

Buteau, C., Muller, E., Marshall, N., Sacristán, A. I., \& Mgombelo, J. (2016). Undergraduate Mathematics Students Appropriating Programming as a Tool for Modelling, Simulation, and Visualization: A Case Study. Digital Experiences in Mathematics Education, 2(2), 142-166. https://doi.org/10.1007/s40751-016-0017-5 
DeJarnette, A. F. (2019). Students' Challenges with Symbols and Diagrams when Using a Programming Environment in Mathematics. Digital Experiences in Mathematics Education, 5(1), 36-58. https://doi.org/10.1007/s40751-018-0044-5

diSessa, A. A. (2017). Conceptual Change in a Microcosm: Comparative Learning Analysis of a Learning Event. Human Development, 60(1), 1-37. https://doi.org/10.1159/000469693

diSessa, A. A. (2018). Computational Literacy and "The Big Picture" Concerning Computers in Mathematics Education. Mathematical Thinking and Learning: An International Journal, 20(1), 3-31. https://doi.org/10.1080/10986065.2018.1403544

Ellis, A., Tillema, E., Lockwood, E., \& Moore, K. (2017). Generalization across Domains: The RelatingForming-Extending Generalization Framework. https://scholarworks.iupui.edu/handle/1805/18132

Evans, J. (1999). Building Bridges: Reflections on the Problem of Transfer of Learning in Mathematics. Educational Studies in Mathematics, 39(1/3), 23-44.

Gibson, J. J. (1979). The Ecological Approach to Visual Perception. Psychology Press.

Gravemeijer, K., Stephan, M., Julie, C., Lin, F.-L., \& Ohtani, M. (2017). What Mathematics Education May Prepare Students for the Society of the Future? International Journal of Science and Mathematics Education, 15(1), 105-123. https://doi.org/10.1007/s10763-017-9814-6

Greeno, J. G., Moore, J. L., \& Smith, D. R. (1993). Transfer of situated learning. In Transfer on trial: Intelligence, cognition, and instruction (pp. 99-167). Ablex Publishing.

Høffding, H. (1892). Outlines of Psychology. Macmillan and Company, Limited.

Inagaki, K., \& Hatano, G. (2002). Young Children's Naive Thinking about the Biological World. Psychology Press.

Karakok, G. (2019). Making connections among representations of eigenvector: What sort of a beast is it? ZDM, 51(7), 1141-1152. https://doi.org/10.1007/s11858-019-01061-9

Kaufmann, O. T., \& Stenseth, B. (2020). Programming in mathematics education. International Journal of Mathematical Education in Science and Technology, O(0), 1-20. https://doi.org/10.1080/0020739X.2020.1736349

Lavy, I. (2006). A case study of different types of arguments emerging from explorations in an interactive computerized environment. The Journal of Mathematical Behavior, 25(2), 153-169. https://doi.org/10.1016/j.jmathb.2006.02.006

Lobato, J. (2008). When students don't apply the knowledge you think they have, rethink your assumptions about transfer (pp. 289-304). https://doi.org/10.5948/UPO9780883859759.023

Lobato, J. (2012). The Actor-Oriented Transfer Perspective and Its Contributions to Educational Research and Practice. Educational Psychologist, 47(3), 232-247. https://doi.org/10.1080/00461520.2012.693353

Lobato, J., \& Siebert, D. (2002). Quantitative reasoning in a reconceived view of transfer. The Journal of Mathematical Behavior, 21(1), 87-116. https://doi.org/10.1016/S0732-3123(02)00105-0

Lockwood, E. (2011). Student connections among counting problems: An exploration using actororiented transfer. Educational Studies in Mathematics, 78. https://doi.org/10.1007/s10649-0119320-7 
Lockwood, E., \& De Chenne, A. (2020). Enriching Students' Combinatorial Reasoning through the Use of Loops and Conditional Statements in Python. International Journal of Research in Undergraduate Mathematics Education, 6(3), 303-346. https://doi.org/10.1007/s40753-01900108-2

Lockwood, E., \& Mørken, K. (2021). A Call for Research that Explores Relationships between Computing and Mathematical Thinking and Activity in RUME. International Journal of Research in Undergraduate Mathematics Education. https://doi.org/10.1007/s40753-020-00129$\underline{2}$

Mørken, K. (n.d.). MAT-INF1100 - Modelling and Computations-Universitetet $i$ Oslo. Retrieved January 29, 2021, from https://www.uio.no/studier/emner/matnat/math/MAT-INF1100/indexeng.html

Mørken, K. (2017). Numerical Algorithms and Digital Representation. https://www.uio.no/studier/emner/matnat/math/MAT-INF1100/h17/kompendiet/matinf1100.pdf

Nowell, L. S., Norris, J. M., White, D. E., \& Moules, N. J. (2017). Thematic Analysis: Striving to Meet the Trustworthiness Criteria. International Journal of Qualitative Methods, 16(1), 1609406917733847. https://doi.org/10.1177/1609406917733847

Papert, S. A. (1993). Mindstorms: Children, Computers, And Powerful Ideas (2 edition). Basic Books.

Passey, D. (2017). Computer science (CS) in the compulsory education curriculum: Implications for future research. Education and Information Technologies, 22(2), 421-443. https://doi.org/10.1007/s10639-016-9475-z

Ramler, I. P., \& Chapman, J. L. (2011). Introducing Statistical Research to Undergraduate Mathematical Statistics Students Using the Guitar Hero Video Game Series. Journal of Statistics Education, 19(3). https://doi.org/10.1080/10691898.2011.11889623

Sand, O. P. (2021). Integrating Computing with Mathematics and Science Education: Case Studies of Student Understanding and Teaching Design [Universitetet i Oslo]. https://www.duo.uio.no/handle/10852/88987

Sand, O. P., Mørken, K., Caballero, M. D., \& Lockwood, E. (2021). Students' Development of a Logarithm Function in Python Using Taylor Expansions: A Teaching Design Case Study [Unpublished].

Sherin, B. L. (2001). A Comparison of Programming Languages and Algebraic Notation as Expressive Languages for Physics. International Journal of Computers for Mathematical Learning, 6(1), 161. https://doi.org/10.1023/A:1011434026437

Weintrop, D., Beheshti, E., Horn, M., Orton, K., Jona, K., Trouille, L., \& Wilensky, U. (2016). Defining Computational Thinking for Mathematics and Science Classrooms. Journal of Science Education and Technology, 25(1), 127-147. https://doi.org/10.1007/s10956-015-9581-5

Williams, J., \& Wake, G. (2007). Metaphors and Models in Translation Between College and Workplace Mathematics. Educational Studies in Mathematics, 64(3), 345-371.

Wolfram Alpha. (n.d.). Retrieved November 26, 2020, from https://www.wolframalpha.com/input/?i=10\%5E10+\%3D+\%28n\%2B1\%29*3\%5E\%28n\%2B1\% $\underline{29}$ 